\title{
On Matsaev's conjecture for contractions on noncommutative $L^{p}$-spaces
}

\author{
Cédric Arhancet
}

\begin{abstract}
We exhibit large classes of contractions on noncommutative $L^{p}$-spaces which satisfy the noncommutative analogue of Matsaev's conjecture, introduced by Peller, in 1985. In particular, we prove that every Schur multiplier on a Schatten space $S^{p}$ induced by a contractive Schur multiplier on $B\left(\ell^{2}\right)$ associated with a real matrix satisfy this conjecture. Moreover, we deal with analogue questions for $C_{0}$-semigroups. Finally, we disprove a conjecture of Peller concerning norms on the space of complex polynomials arising from Matsaev's conjecture and Peller's problem. Indeed, if $S$ denotes the shift on $\ell^{p}$ and $\sigma$ the shift on the Schatten space $S^{p}$, the norms $\|P(S)\|_{\ell^{p} \rightarrow \ell^{p}}$ and $\left\|P(\sigma) \otimes \operatorname{Id}_{S^{p}}\right\|_{S^{p}\left(S^{p}\right) \rightarrow S^{p}\left(S^{p}\right)}$ can be different for a complex polynomial $P$.
\end{abstract}

\section{Introduction}

To estimate the norms of functions of operators is an essential task in Operator Theory. In this subject, V. V. Matsaev stated the following conjecture in 1971, see Nik1. For any $1 \leqslant p \leqslant \infty$, let $\ell^{p} \stackrel{S}{\rightarrow} \ell^{p}$ denote the right shift operator defined by $S\left(a_{0}, a_{1}, a_{2}, \ldots\right)=\left(0, a_{0}, a_{1}, a_{2}, \ldots\right)$.

Conjecture 1.1 Suppose $1<p<\infty, p \neq 2$. Let $\Omega$ be a measure space and let $L^{p}(\Omega) \stackrel{T}{\rightarrow} L^{p}(\Omega)$ be a contraction. For any complex polynomial $P$, we have

$$
\|P(T)\|_{L^{p}(\Omega) \rightarrow L^{p}(\Omega)} \leqslant\|P(S)\|_{\ell^{p} \rightarrow \ell^{p}} .
$$

It is easy to see that (1.1) holds true for $p=1$ and $p=\infty$. Moreover, by using the Fourier transform, it is clear that for $p=2$, (1.1) is a consequence of von Neumann's inequality. Finally, very recently and after the writing of this paper, S. W. Drury $[\mathrm{Dr}]$ found a counterexample in the case $p=4$ by using computer.

For all other values of $p$, the validity of (1.1) for any contraction is open. It is well-known that (1.1) holds true for any positive contraction, more generally for all operators $L^{p}(\Omega) \stackrel{T}{\rightarrow} L^{p}(\Omega)$ which admit a contractive majorant (i.e. there exists a positive contraction $\tilde{T}$ satisfying $|T(f)| \leqslant \tilde{T}(|f|)$ ). This follows from the fact that these operators admit an isometric dilation. We refer the reader to [ALM], [AkS], [CoW], [Kit], [Nik2] and [Pel1] for information and historical background on this question.

In 1985, V.V. Peller Pel2 introduced a noncommutative version of Matsaev's conjecture for Schatten spaces $S^{p}=S^{p}\left(\ell^{2}\right)$. Recall that elements of $S^{p}$ can be regarded as infinite matrices indexed by

This work is partially supported by ANR 06-BLAN-0015.

2000 Mathematics subject classification: Primary 46L51; Secondary, 46M35, 46L07

Key words and phrases: Matsaev's conjecture, noncommutative $L_{p}$-spaces, complex interpolation, Schur multipliers, Fourier multipliers, dilations, semigroups. 
$\mathbb{N} \times \mathbb{N}$. Thus we define the linear map $S^{p} \stackrel{\sigma}{\rightarrow} S^{p}$ as the shift 'from NW to SE' which maps any matrix

$$
\left[\begin{array}{cccc}
a_{00} & a_{01} & a_{02} & \cdots \\
a_{10} & a_{11} & a_{12} & \cdots \\
a_{20} & a_{21} & a_{22} & \cdots \\
\cdots & \cdots & \cdots & \cdots
\end{array}\right] \text { to }\left[\begin{array}{cccc}
0 & 0 & 0 & \cdots \\
0 & a_{00} & a_{01} & \cdots \\
0 & a_{10} & a_{11} & \cdots \\
\cdots & \cdots & \cdots & \cdots
\end{array}\right] \text {. }
$$

Let $S^{p}\left(S^{p}\right)$ be the space of all matrices $\left[a_{i j}\right]_{i, j \geqslant 0}$ with entries $a_{i j}$ in $S^{p}$, which represent an element of the bigger Schatten space $S^{p}\left(\ell^{2} \otimes_{2} \ell^{2}\right)$. The algebraic tensor product $S^{p} \otimes S^{p}$ can be regarded as a dense subspace of $S^{p}\left(S^{p}\right)$ in a natural way. Then the mapping on $S^{p}\left(S^{p}\right)$ given by (1.2) is an isometry, which is the unique extension of $\sigma \otimes I_{S^{p}}$ to the space $S^{p}\left(S^{p}\right)$. (See Section 2 below for more details on these matricial representations.) Peller's question is as follows.

Question 1.2 Suppose $1<p<\infty, p \neq 2$. Let $S^{p} \stackrel{T}{\rightarrow} S^{p}$ be a contraction on the Schatten space $S^{p}$. Do we have

$$
\|P(T)\|_{S^{p} \rightarrow S^{p}} \leqslant\left\|P(\sigma) \otimes I d_{S^{p}}\right\|_{S^{p}\left(S^{p}\right) \rightarrow S^{p}\left(S^{p}\right)}
$$

for any complex polynomial $P$ ?

Peller observed that (1.3) holds true when $T$ is an isometry or when $S^{p} \stackrel{T}{\rightarrow} S^{p}$ is defined by $T(x)=a x b$, where $\ell^{2} \stackrel{a}{\rightarrow} \ell^{2}$ and $\ell^{2} \stackrel{b}{\rightarrow} \ell^{2}$ are contractions.

The Schatten spaces $S^{p}$ are basic examples of noncommutative $L^{p}$-spaces. It is then natural to extend Peller's problem to this wider context. This leads to the following question.

Question 1.3 Suppose $1<p<\infty, p \neq 2$. Let $M$ be a semifinite von Neumann algebra and let $L^{p}(M)$ be the associated noncommutative $L^{p}$-space. Let $L^{p}(M) \stackrel{T}{\rightarrow} L^{p}(M)$ be a contraction. Do we have

$$
\|P(T)\|_{L^{p}(M) \rightarrow L^{p}(M)} \leqslant\left\|P(\sigma) \otimes I d_{S^{p}}\right\|_{S^{p}\left(S^{p}\right) \rightarrow S^{p}\left(S^{p}\right)}
$$

for any complex polynomial $P$ ?

As in the commutative case, it is easy to see that (1.4) holds true when $p=1, p=2$ or $p=\infty$. The main purpose of this article is to exhibit large classes of contractions on noncommutative $L^{p}$-spaces which satisfy inequality (1.4) for any complex polynomial $P$. The next theorem gathers some of our main results.

Theorem 1.4 Suppose $1<p<\infty$. The following maps satisfy (1.4) for any complex polynomial $P$.

1. A Schur multiplier $S^{p} \stackrel{M_{A}}{\longrightarrow} S^{p}$ induced by a contractive Schur multiplier $B\left(\ell^{2}\right) \stackrel{M_{A}}{\longrightarrow} B\left(\ell^{2}\right)$ associated with a real-valued matrix $A$.

2. A Fourier multiplier $L^{p}(\mathrm{VN}(G)) \stackrel{M_{t}}{\longrightarrow} L^{p}(\mathrm{VN}(G))$ induced by a contractive Fourier multiplier $\mathrm{VN}(G) \stackrel{M_{t}}{\longrightarrow} \mathrm{VN}(G)$ associated with a real valued function $G \stackrel{t}{\rightarrow} \mathbb{R}$, in the case where $G$ is an amenable discrete group $G$.

3. A Fourier multiplier $L^{p}\left(\mathrm{VN}\left(\mathbb{F}_{n}\right)\right) \stackrel{M_{t}}{\longrightarrow} L^{p}\left(\mathrm{VN}\left(\mathbb{F}_{n}\right)\right)$ induced by a unital completely positive Fourier multiplier $\mathrm{VN}\left(\mathbb{F}_{n}\right) \stackrel{M_{t}}{\longrightarrow} \mathrm{VN}\left(\mathbb{F}_{n}\right)$ associated with a real valued function $\mathbb{F}_{n} \stackrel{t}{\rightarrow} \mathbb{R}$, where $\mathbb{F}_{n}$ is the free group with $n$ generators $(1 \leqslant n \leqslant \infty)$. 
The proof of these results will use dilation theorems that we now state. Moreover, these theorems rely on constructions dues to É. Ricard [Ric].

Theorem 1.5 Let $B\left(\ell^{2}\right) \stackrel{M_{A}}{\longrightarrow} B\left(\ell^{2}\right)$ be a unital completely positive Schur multiplier with a real-valued matrix $A$. Then there exists a hyperfinite von Neumann algebra $M$ equipped with a semifinite normal faithful trace, a unital trace preserving *-automorphism $M \stackrel{U}{\rightarrow} M$, a unital trace preserving one-to-one normal *-homomorphism $B\left(\ell^{2}\right) \stackrel{J}{\rightarrow} M$ such that

$$
\left(M_{A}\right)^{k}=\mathbb{E} U^{k} J
$$

for any integer $k \geqslant 0$, where $M \stackrel{\mathbb{E}}{\rightarrow} B\left(\ell^{2}\right)$ is the canonical faithful normal trace preserving conditional expectation associated with $J$.

Theorem 1.6 Let $G$ be a discrete group. Let $\mathrm{VN}(G) \stackrel{M_{t}}{\longrightarrow} \mathrm{VN}(G)$ be a unital completely positive Fourier multiplier associated with a real valued function $G \stackrel{t}{\rightarrow} \mathbb{R}$. Then there exists a von Neumann algebra $M$ equipped with a faithful finite normal trace, a unital trace preserving *-automorphism $M \stackrel{U}{\rightarrow}$ $M$, a unital normal trace preserving one-to-one $*$-homomorphism $\mathrm{VN}(G) \stackrel{J}{\rightarrow} M$ such that,

$$
\left(M_{t}\right)^{k}=\mathbb{E} U^{k} J
$$

for any integer $k \geqslant 0$, where $M \stackrel{\mathbb{E}}{\rightarrow} V N(G)$ is the canonical faithful normal trace preserving conditional expectation associated with $J$. Moreover, if $G$ is amenable or if $G=\mathbb{F}_{n}(1 \leqslant n \leqslant \infty)$, the von Neumann algebra $M$ has the quotient weak expectation property.

Various norms on the space of complex polynomials arise from Matsaev's conjecture and Peller's problem, and it is interesting to try to compare them. If $1 \leqslant p \leqslant \infty$, note that the space of all diagonal matrices in $S^{p}$ can be identified with $\ell^{p}$. In this regard, the shift operator $\ell^{p} \stackrel{S}{\rightarrow} \ell^{p}$ coincides with the restriction of $S^{p} \stackrel{\sigma}{\rightarrow} S^{p}$ to diagonal matrices. This readily implies that

$$
\|P(S)\|_{\ell^{p} \rightarrow \ell^{p}} \leqslant\|P(\sigma)\|_{S^{p} \rightarrow S^{p}} \leqslant\left\|P(\sigma) \otimes I d_{S^{p}}\right\|_{S^{p}\left(S^{p}\right) \rightarrow S^{p}\left(S^{p}\right)}
$$

for any complex polynomial $P$. We will show the following result, which disproves a conjecture due to Peller [Pel2, Conjecture 2].

Theorem 1.7 Suppose $1<p<\infty, p \neq 2$. Then there exists a complex polynomial $P$ such that

$$
\|P(S)\|_{\ell^{p} \rightarrow \ell^{p}}<\left\|P(\sigma) \otimes I d_{S^{p}}\right\|_{S^{p}\left(S^{p}\right) \rightarrow S^{p}\left(S^{p}\right)} .
$$

To complete this investigation, we will also show that

$$
\|P(\sigma)\|_{S^{p} \rightarrow S^{p}}=\left\|P(\sigma) \otimes I d_{S^{p}}\right\|_{S^{p}\left(S^{p}\right) \rightarrow S^{p}\left(S^{p}\right)}=\left\|P(S) \otimes I d_{S^{p}}\right\|_{\ell^{p}\left(S^{p}\right) \rightarrow \ell^{p}\left(S^{p}\right)}
$$

for any $P$ (the first of these equalities being due to É. Ricard).

The paper is organized as follows. In $\S 2$, we fix some notations, we give some background on the key notion of completely bounded maps on noncommutative $L^{p}$-spaces, we prove the second equality of (1.5) and we give some preliminary results. In $\S 3$, we show that some Fourier multipliers on $L^{p}(\mathbb{R})$ and $\ell_{\mathbb{Z}}^{p}$ are bounded but not completely bounded and we prove Theorem [1.7 and the first equality of (1.5). $\S 4$ is devoted to classes of contractions which satisfy noncommutative Matsaev's inequality (1.4) for any complex polynomial $P$. In particular we prove Theorems 1.5 and 1.6. In $\S 5$, we consider a natural analog of Question 1.3 for $C_{0}$-semigroups of contractions. Finally in $\S 6$, we exhibit some polynomials $P$ which always satisfy (1.4) for any contraction $T$. 


\section{Preliminaries}

Let us recall some basic notations. Let $\mathbb{T}=\{z \in \mathbb{C}|| z \mid=1\}$ and $\delta_{i, j}$ the symbol of Kronecker.

If $I$ is an index set and if $E$ is a vector space, we write $\mathbb{M}_{I}$ for the space of the $I \times I$ matrices with entries in $\mathbb{C}$ and $\mathbb{M}_{I}(E)$ for the space of the $I \times I$ matrices with entries in $E$. If $K$ is another index set, we have an isomorphism $\mathbb{M}_{I}\left(\mathbb{M}_{K}\right)=\mathbb{M}_{I \times K}$.

Let $M$ be a von Neumann algebra equipped with a semifinite normal faithful trace $\tau$. For $1 \leqslant p<\infty$ the noncommutative $L^{p}$-space $L^{p}(M)$ is defined as follows. If $S^{+}$is the set of all positive $x \in M$ such that $\tau(x)<\infty$ and $S$ is its linear span, then $L^{p}(M)$ is the completion of $S$ with respect to the norm $\|x\|_{L^{p}(M)}=\tau\left(|x|^{p}\right)^{\frac{1}{p}}$. One sets $L^{\infty}(M)=M$. We refer to [PiX], and the references therein, for more information on these spaces.

Let $1 \leqslant p<\infty$. If $I$ is an index set and if we equip the space $B\left(\ell_{I}^{2}\right)$ with the operator norm and the canonical trace $\operatorname{Tr}$, the space $L^{p}\left(B\left(\ell_{I}^{2}\right)\right)$ identifies to the Schatten-von Neumann class $S_{I}^{p}$. The space $S_{I}^{p}$ is the space of those compact operators $x$ from $\ell_{I}^{2}$ into $\ell_{I}^{2}$ such that $\|x\|_{S_{I}^{p}}=\left(\operatorname{Tr}\left(x^{*} x\right)^{\frac{p}{2}}\right)^{\frac{1}{p}}<\infty$. The space $S_{I}^{\infty}$ of compact operators from $\ell_{I}^{2}$ into $\ell_{I}^{2}$ is equipped with the operator norm. For $I=\mathbb{N}$, we simplify the notations, we let $S^{p}$ for $S_{\mathbb{N}}^{p}$. Elements of $S_{I}^{p}$ are regarded as matrices $A=\left[a_{i j}\right]_{i, j \in I}$ of $\mathbb{M}_{I}$. The space $S_{I}^{p}\left(S_{K}^{p}\right)$ is the space of those compact operators $x$ from $\ell_{I}^{2} \otimes_{2} \ell_{K}^{2}$ into $\ell_{I}^{2} \otimes_{2} \ell_{K}^{2}$ such that $\|x\|_{S_{I}^{p}\left(S_{K}^{p}\right)}=\left((\operatorname{Tr} \otimes \operatorname{Tr})\left(x^{*} x\right)^{\frac{p}{2}}\right)^{\frac{1}{p}}<\infty$. Elements of $S_{I}^{p}\left(S_{K}^{p}\right)$ are regarded as matrices of $\mathbb{M}_{I}\left(\mathbb{M}_{K}\right)$.

Let $M$ be a von Neumann algebra equipped with a semifinite normal faithful trace $\tau$. If the von Neumann algebra $B\left(\ell_{I}^{2}\right) \bar{\otimes} M$ is equipped with the semifinite normal faithful trace $\operatorname{Tr} \otimes \tau$, the space $L^{p}\left(B\left(\ell_{I}^{2}\right) \bar{\otimes} M\right)$ identifies to a space $S_{I}^{p}\left(L^{p}(M)\right)$ of matrices of $\mathbb{M}_{I}\left(L^{p}(M)\right)$. Moreover, under this identification, the algebraic tensor product $S_{I}^{p} \otimes L^{p}(M)$ is dense in $S_{I}^{p}\left(L^{p}(M)\right)$.

Let $N$ be another von Neumann algebra equipped with a semifinite normal faithful trace. If $1 \leqslant p \leqslant \infty$, we say that a linear map $L^{p}(M) \stackrel{T}{\rightarrow} L^{p}(N)$ is completely bounded if $I d_{S^{p}} \otimes T$ extends to a bounded operator $S^{p}\left(L^{p}(M)\right) \stackrel{I d_{S^{p}} \otimes T}{\longrightarrow} S^{p}\left(L^{p}(N)\right)$. In this case, the completely bounded norm $\|T\|_{c b, L^{p}(M) \rightarrow L^{p}(N)}$ is defined by

$$
\|T\|_{c b, L^{p}(M) \rightarrow L^{p}(N)}=\left\|I d_{S^{p}} \otimes T\right\|_{S^{p}\left(L^{p}(M)\right) \rightarrow S^{p}\left(L^{p}(N)\right)} .
$$

If $\Omega$ is a measure space, the space $S^{p}\left(L^{p}(\Omega)\right)$ is isometric to the $L^{p}$-space $L^{p}\left(\Omega, S^{p}\right)$ of $S^{p}$-valued functions in Bochner's sense. Thus, if $L^{p}(\Omega) \stackrel{T}{\rightarrow} L^{p}(\Omega)$ is a linear map, we have

$$
\|T\|_{c b, L^{p}(\Omega) \rightarrow L^{p}(\Omega)}=\left\|T \otimes I d_{S^{p}}\right\|_{L^{p}\left(\Omega, S^{p}\right) \rightarrow L^{p}\left(\Omega, S^{p}\right)} .
$$

The notion of completely bounded map and the completely bounded norm defined in (2.1) are the same that these defined in operator space theory, see [EfR], [Pis2] and [Pis4].

Now, we let:

Definition 2.1 Let $M$ be a von Neumann algebra equipped with a faithful semifinite normal trace and $1 \leqslant p \leqslant \infty$. Let $L^{p}(M) \stackrel{T}{\rightarrow} L^{p}(M)$ be a contraction. We say that $T$ satisfies the noncommutative Matsaev's property if (1.4) holds for any complex polynomial $P$.

We denote by $\ell^{p} \stackrel{S}{\rightarrow} \ell^{p}$ the right shift on $\ell^{p}$. We use the same notation for the right shift on $\ell_{\mathbb{Z}}^{p}$. We denote by $S_{-}$the left shift on $\ell^{p}$ defined by $S_{-}\left(a_{0}, a_{1}, a_{2}, \ldots\right)=\left(a_{1}, a_{2}, a_{3}, \ldots\right)$. Suppose $1 \leqslant p \leqslant \infty$. Let $X$ be a Banach space. For any complex polynomial $P$, we define $\|P\|_{p, X}$ by

$$
\|P\|_{p, X}=\left\|P(S) \otimes I d_{X}\right\|_{\ell^{p}(X) \rightarrow \ell^{p}(X)} .
$$


We let $\|P\|_{p}=\|P\|_{p, \mathbb{C}}\left(=\|P(S)\|_{\ell^{p} \rightarrow \ell^{p}}\right)$. If $1 \leqslant p<\infty$, it is easy to see that, for any complex polynomial $P$, we have

$$
\|P\|_{p, X}=\left\|P(S) \otimes I d_{X}\right\|_{\ell_{\mathbb{Z}}^{p}(X) \rightarrow \ell_{\mathbb{Z}}^{p}(X)}=\left\|P\left(S_{-}\right) \otimes I d_{X}\right\|_{\ell^{p}(X) \rightarrow \ell^{p}(X)} .
$$

Moreover, for all $1 \leqslant p<\infty$, by (2.2), we have

$$
\|P\|_{p, S^{p}}=\|P(S)\|_{c b, \ell_{\mathbb{Z}}^{p} \rightarrow \ell_{\mathbb{Z}}^{p}}
$$

Note that, if $1 \leqslant p \leqslant \infty$, we have $\|P\|_{p, S^{p}}=\|P\|_{p^{*}, S_{p^{*}}}$. Moreover, if $1 \leqslant p \leqslant q \leqslant 2$, we have $\|P\|_{q, S_{q}} \leqslant\|P\|_{p, S^{p}}$ by interpolation. We define the linear map $S_{\mathbb{Z}}^{p} \stackrel{\Theta}{\rightarrow} S_{\mathbb{Z}}^{p}$ as the shift "from NW to SE" which maps any matrix

$$
\left[\begin{array}{ccccc}
\cdots & \cdots & \cdots & \cdots & \cdots \\
\cdots & a_{0,0} & a_{0,1} & a_{0,2} & \cdots \\
\cdots & a_{1,0} & a_{1,1} & a_{1,2} & \cdots \\
\cdots & a_{2,0} & a_{2,1} & a_{2,2} & \cdots \\
\cdots & \cdots & \cdots & \cdots & \cdots
\end{array}\right] \text { to }\left[\begin{array}{ccccc}
\cdots & \cdots & \cdots & \cdots & \cdots \\
\cdots & a_{-1,-1} & a_{-1,0} & a_{-1,1} & \cdots \\
\cdots & a_{0,-1} & a_{0,0} & a_{0,1} & \cdots \\
\cdots & a_{1,-1} & a_{1,0} & a_{1,1} & \cdots \\
\cdots & \cdots & \cdots & \cdots & \cdots
\end{array}\right]
$$

If $1 \leqslant p<\infty$, it is not difficult to see that for any complex polynomial $P$ we have

$$
\|P(\Theta)\|_{S_{\mathbb{Z}}^{p} \rightarrow S_{Z}^{p}}=\|P(\sigma)\|_{S^{p} \rightarrow S^{p}} \quad \text { and } \quad\|P(\Theta)\|_{c b, S_{Z}^{p} \rightarrow S_{Z}^{p}}=\|P(\sigma)\|_{c b, S^{p} \rightarrow S^{p}} .
$$

Moreover, it is easy to see that, for all $A \in S_{\mathbb{Z}}^{p}$, we have the equality $\Theta(A)=S A S^{-1}$ where we consider $A$ and $\Theta(A)$ as operators on $\ell_{\mathbb{Z}}^{2}$.

We will use the following theorem inspired by a well-known technique of Kitover.

Theorem 2.2 Suppose $1 \leqslant p \leqslant \infty$. Let $X$ be a Banach space and $X \stackrel{T}{\rightarrow} X$ an isometry (not necessarily onto). For any complex polynomial $P$, we have the inequality

$$
\|P(T)\|_{X \rightarrow X} \leqslant\|P\|_{p, X} .
$$

Proof : It suffices to consider the case $1<p<\infty$. Let $0<r<1$. Since $T$ is an isometry we have

$$
\sum_{j=0}^{+\infty}\left\|r^{j} T^{j}(x)\right\|_{X}^{p}=\sum_{j=0}^{+\infty} r^{j p}\left\|T^{j}(x)\right\|_{X}^{p}=\|x\|_{X}^{p}\left(\sum_{j=0}^{+\infty}\left(r^{p}\right)^{j}\right)<+\infty
$$

We let $C_{r}=\left(\sum_{j=0}^{+\infty} r^{j p}\right)^{\frac{1}{p}}$. Now we define the operator

$$
\begin{array}{ccc}
W_{r}: X & \longrightarrow & \ell^{p}(X) \\
x & \longmapsto \frac{1}{C_{r}}\left(x, r T(x), r^{2} T^{2}(x), \ldots, r^{j} T^{j}(x), \ldots\right)
\end{array}
$$

which is an isometry. If $n$ is a positive integer and if $x \in X$ we have

$$
W_{r}\left((r T)^{n} x\right)=\frac{1}{C_{r}}\left(r^{n} T^{n} x, r^{n+1} T^{n+1} x, \ldots\right)=\left(S_{-} \otimes I d_{X}\right)^{n}\left(W_{r}(x)\right) .
$$


We deduce that for any complex polynomial $P$ we have $W_{r} P(r T)=P\left(S_{-} \otimes I d_{X}\right) W_{r}$. Now, if $x \in X$, we have

$$
\begin{aligned}
\|P(r T) x\|_{X} & =\left\|W_{r}(P(r T) x)\right\|_{\ell^{p}(X)} \\
& =\left\|P\left(S_{-} \otimes I d_{X}\right) W_{r}(x)\right\|_{\ell^{p}(X)} \\
& \leqslant\left\|P\left(S_{-}\right) \otimes I d_{X}\right\|_{\ell^{p}(X) \rightarrow \ell^{p}(X)}\|x\|_{X} \\
& =\|P\|_{p, X}\|x\|_{X} \quad \text { by (2.3). }
\end{aligned}
$$

Consequently, letting $r$ to 1 , we obtain finally that $\|P(T)\|_{X \rightarrow X} \leqslant\|P\|_{p, X}$.

Corollary 2.3 Suppose $1 \leqslant p \leqslant \infty$. Let $P$ be a complex polynomial. We have

$$
\left\|P(\sigma) \otimes I d_{S^{p}}\right\|_{S^{p}\left(S^{p}\right) \rightarrow S^{p}\left(S^{p}\right)}=\|P\|_{p, S^{p}} .
$$

Proof : With the diagonal embedding of $\ell^{p}$ in $S^{p}$, we see that for any complex polynomial $P$ we have

$$
\|P\|_{p, S^{p}} \leqslant\left\|P(\sigma) \otimes I d_{S^{p}}\right\|_{S^{p}\left(S^{p}\right) \rightarrow S^{p}\left(S^{p}\right)} .
$$

Now the map $S^{p}\left(S^{p}\right) \stackrel{\sigma \otimes I d_{S^{p}}}{\longrightarrow} S^{p}\left(S^{p}\right)$ is an isometry. Hence, by the above theorem, we deduce that for every complex polynomial $P$ we have

$$
\left\|P(\sigma) \otimes I d_{S^{p}}\right\|_{S^{p}\left(S^{p}\right) \rightarrow S^{p}\left(S^{p}\right)}=\left\|P\left(\sigma \otimes I d_{S^{p}}\right)\right\|_{S^{p}\left(S^{p}\right) \rightarrow S^{p}\left(S^{p}\right)} \leqslant\|P\|_{p, S^{p}\left(S^{p}\right)}=\|P\|_{p, S^{p}} .
$$

Let $M$ be a von Neumann algebra. Let us recall that $M$ has QWEP means that $M$ is the quotient of a $C^{*}$-algebra having the weak expectation property (WEP) of C. Lance (see Oza for more information on these notions). It is unknown whether every von Neumann algebra has this property. We will need the following theorem which is a particular case of a result of Jun].

Theorem 2.4 Let $M$ be a von Neumann algebra with QWEP equipped with a faithful semifinite normal trace. Suppose $1<p<\infty$. Let $\Omega$ be a measure space. Suppose that $L^{p}(\Omega) \stackrel{T}{\rightarrow} L^{p}(\Omega)$ is a completely bounded map. Then $T \otimes I d_{L^{p}(M)}$ extends to a bounded operator and we have

$$
\left\|T \otimes I d_{L^{p}(M)}\right\|_{L^{p}\left(\Omega, L^{p}(M)\right) \rightarrow L^{p}\left(\Omega, L^{p}(M)\right)} \leqslant\|T\|_{c b, L^{p}(\Omega) \rightarrow L^{p}(\Omega)} .
$$

In the case where $M$ is a hyperfinite von Neumann algebra, the statement of this theorem is easy to prove (use [Pis2, (3.1)] and [Pis2, (3.6)]). With this theorem, we deduce the following proposition.

Proposition 2.5 Suppose $1<p<\infty$. Let $M$ be a von Neumann algebra with QWEP equipped with a faithful semifinite normal trace. For all complex polynomial $P$ we have

$$
\|P\|_{p, L^{p}(M)} \leqslant\|P\|_{p, S^{p}} .
$$

With this proposition, we can prove the following corollary.

Corollary 2.6 Let $M$ be a von Neumann algebra equipped with a faithful semifinite normal trace and $1<p<\infty$. Let $L^{p}(M) \stackrel{T}{\rightarrow} L^{p}(M)$ be a contraction. Suppose that there exists a von Neumann algebra $M$ with $Q W E P$ equipped with a faithful semifinite normal trace, an isometric embedding $L^{p}(M) \stackrel{J}{\rightarrow}$ $L^{p}(N)$, an isometry $L^{p}(N) \stackrel{U}{\rightarrow} L^{p}(N)$ and a contractive projection $L^{p}(N) \stackrel{Q}{\rightarrow} L^{p}(M)$ such that,

$$
T^{k}=Q U^{k} J
$$

for any integer $k \geqslant 0$. Then the contraction $T$ has the noncommutative Matsaev's property. 
Proof : For any complex polynomial $P$, we have

$$
\begin{aligned}
\|P(T)\|_{L^{p}(M) \rightarrow L^{p}(M)} & =\|Q P(U) J\|_{L^{p}(M) \rightarrow L^{p}(M)} \\
& \leqslant\|P(U)\|_{L^{p}(N) \rightarrow L^{p}(N)}
\end{aligned}
$$

By using Theorem 2.2] we obtain the inequality

$$
\|P(T)\|_{L^{p}(M) \rightarrow L^{p}(M)} \leqslant\|P\|_{p, L^{p}(N)} .
$$

Now, the von Neumann algebra $N$ is QWEP. Then, by Proposition 2.5] we obtain finally that

$$
\|P(T)\|_{L^{p}(M) \rightarrow L^{p}(M)} \leqslant\|P\|_{p, S^{p}} .
$$

Theorem 2.4. Proposition 2.5 and Corollary 2.6 hold true more generally for noncommutative $L^{p}$-spaces of a von Neumann algebra equipped with a distinguished normal faithful state $M \stackrel{\varphi}{\rightarrow} \mathbb{C}$, constructed by Haagerup. See [PiX] and the references therein for more informations on these spaces.

We refer to [ALM], [AkS], [JLM] and [Pel1] for information on dilations on $L^{p}$-spaces (commutative and noncommutative).

\section{Comparison between the commutative and noncommutative cases}

Suppose $1<p<\infty$. Let $G$ be a locally compact abelian group with dual group $\widehat{G}$. An operator $L^{p}(G) \stackrel{T}{\rightarrow} L^{p}(G)$ is a Fourier multiplier if there exists a function $\psi \in L^{\infty}(\widehat{G})$ such that for any $f \in L^{p}(G) \cap L^{2}(G)$ we have $\mathcal{F}(T(f))=\psi \mathcal{F}(f)$ where $\mathcal{F}$ denotes the Fourier transform. In this case, we let $T=M_{\psi}$. G. Pisier showed that, if $G$ is a compact group and $1<p<\infty, p \neq 2$, there exists a bounded Fourier multiplier $L^{p}(G) \stackrel{T}{\rightarrow} L^{p}(G)$ which is not completely bounded (see [Pis2, Proposition 8.1.3]. We will show this result is also true for the groups $\mathbb{R}$ and $\mathbb{Z}$ and we will prove Theorem 1.7 .

If $b \in L^{1}(G)$, we define the convolution operator $C_{b}$ by

$$
\begin{aligned}
C_{b}: L^{p}(G) & \longrightarrow L^{p}(G) \\
f & \longmapsto b * f .
\end{aligned}
$$

This operator is a completely bounded Fourier multiplier. We observe that, if $P=\sum_{k=0}^{n} a_{k} z^{k}$ is a complex polynomial, the operator $\ell_{\mathbb{Z}}^{p} \stackrel{P(S)}{\longrightarrow} \ell_{\mathbb{Z}}^{p}$ is the operator $\ell_{\mathbb{Z}}^{p} \stackrel{C_{\tilde{a}}}{\longrightarrow} \ell_{\mathbb{Z}}^{p}$ where $\tilde{a}$ is the sequence defined by $\tilde{a}_{k}=a_{k}$ if $0 \leqslant k \leqslant n$ and $\tilde{a}_{k}=0$ otherwise.

We will use the following approximation result [Lar, Theorem 5.6.1].

Theorem 3.1 Suppose $1 \leqslant p<\infty$. Let $G$ be a locally compact abelian group. Let $L^{p}(G) \stackrel{T}{\rightarrow} L^{p}(G)$ be a bounded Fourier multiplier. Then there exists a net of continuous functions $\left(b_{l}\right)_{i \in L}$ with compact support such that

$$
\left\|C_{b_{l}}\right\|_{L^{p}(G) \rightarrow L^{p}(G)} \leqslant\|T\|_{L^{p}(G) \rightarrow L^{p}(G)} \quad \text { and } \quad C_{b_{l}} \underset{l}{\stackrel{s o}{\longrightarrow}} T
$$

(convergence for the strong operator topology).

Moreover, we need the following vectorial extension of [DeL, Proposition 3.3]. One can prove this theorem as [CoW, Theorem 3.4]. 
Theorem 3.2 Suppose $1<p<\infty$. Let $\psi$ be a continuous function on $\mathbb{R}$ which defines a completely bounded Fourier multiplier $M_{\psi}$ on $L^{p}(\mathbb{R})$. Then the restriction $\psi \mid \mathbb{Z}$ of the function $\psi$ to $\mathbb{Z}$ defines a completely bounded Fourier multiplier $M_{\psi \mid \mathbb{Z}}$ on $L^{p}(\mathbb{T})$.

We will use the next result of Jodeit [Jod, Theorem 3.5]. We introduce the function $\Lambda: \mathbb{R} \rightarrow \mathbb{R}$ defined by

$$
\Lambda(x)=\left\{\begin{array}{cll}
1-|x| & \text { if } & x \in[-1,1] \\
0 & \text { if } & |x|>1
\end{array}\right.
$$

Theorem 3.3 Suppose $1<p<\infty$. Let $\varphi$ be a complex function defined on $\mathbb{Z}$ such that $M_{\varphi}$ is a bounded Fourier multiplier on $L^{p}(\mathbb{T})$. Then the complex function $\mathbb{R} \stackrel{\psi}{\rightarrow} \mathbb{C}$ defined on $\mathbb{R}$ by

$$
\psi(x)=\sum_{k \in \mathbb{Z}} \varphi(k) \Lambda(x-k), \quad x \in \mathbb{R}
$$

defines a bounded Fourier multiplier $L^{p}(\mathbb{R}) \stackrel{M_{\psi}}{\longrightarrow} L^{p}(\mathbb{R})$.

Now, we are ready to prove the following theorem.

Theorem 3.4 Suppose $1<p<\infty, p \neq 2$. Then there exists a bounded Fourier multiplier $L^{p}(\mathbb{R}) \stackrel{M_{\psi}}{\longrightarrow}$ $L^{p}(\mathbb{R})$ which is not completely bounded.

Proof : By [Pis2, Proposition 8.1.3], there exists a bounded Fourier multiplier $L^{p}(\mathbb{T}) \stackrel{M_{\varphi}}{\longrightarrow} L^{p}(\mathbb{T})$ which is not completely bounded. Now, we define the function $\psi$ on $\mathbb{R}$ by (3.1). By Theorem 3.3 , the function $\mathbb{R} \stackrel{\psi}{\rightarrow} \mathbb{C}$ defines a bounded Fourier multiplier $L^{p}(\mathbb{R}) \stackrel{M_{\psi}}{\longrightarrow} L^{p}(\mathbb{R})$. Now, suppose that $M_{\psi}$ is completely bounded. Since the function $\mathbb{R} \stackrel{\psi}{\rightarrow} \mathbb{C}$ is continuous, by Theorem 3.2 , we deduce that the restriction $\psi \mid \mathbb{Z}$ defines a completely bounded Fourier multiplier $M_{\psi \mid \mathbb{Z}}$ on $L^{p}(\mathbb{T})$. Moreover, we observe that, for all $k \in \mathbb{Z}$, we have

$$
\psi(k)=\varphi(k) .
$$

Then we deduce that the Fourier multiplier $L^{p}(\mathbb{T}) \stackrel{M_{\varphi}}{\longrightarrow} L^{p}(\mathbb{T})$ is completely bounded. We obtain a contradiction. Consequently, the bounded Fourier multiplier $L^{p}(\mathbb{R}) \stackrel{M_{\psi}}{\longrightarrow} L^{p}(\mathbb{R})$ is not completely bounded.

The proof of the next theorem is inspired by [CoW, page 25].

Theorem 3.5 Suppose $1<p<\infty, p \neq 2$. Then

1. There exists a bounded Fourier multiplier $\ell_{\mathbb{Z}}^{p} \stackrel{T}{\rightarrow} \ell_{\mathbb{Z}}^{p}$ which is not completely bounded.

2. There exists a complex polynomial $P$ such that $\|P\|_{p}<\|P\|_{p, S^{p}}$.

Proof : By Theorem 3.4 , there exists a bounded Fourier multiplier $L^{p}(\mathbb{R}) \stackrel{M_{\psi}}{\longrightarrow} L^{p}(\mathbb{R})$ which is not completely bounded. We can suppose that $M_{\psi}$ satisfies $\left\|M_{\psi}\right\|_{L^{p}(\mathbb{R}) \rightarrow L^{p}(\mathbb{R})}=1$. By Theorem 3.1 there exists a net of continuous functions $\left(b_{l}\right)_{l \in L}$ with compact support such that

$$
\left\|C_{b_{l}}\right\|_{L^{p}(\mathbb{R}) \rightarrow L^{p}(\mathbb{R})} \leqslant 1 \text { and } \quad C_{b_{l}} \underset{l}{\stackrel{s o}{\longrightarrow}} M_{\psi} .
$$

Let $c>1$. There exists an element $y=\sum_{k=1}^{n} f_{k} \otimes x_{k} \in L^{p}(\mathbb{R}) \otimes S^{p}$ with $\|y\|_{L^{p}\left(\mathbb{R}, S^{p}\right)} \leqslant 1$ such that $\left\|\left(M_{\psi} \otimes I d_{S^{p}}\right)(y)\right\|_{L^{p}\left(\mathbb{R}, S^{p}\right)} \geqslant 3 c$. Then, it is not difficult to see that there exists $l \in L$ such that 
$\left\|\left(C_{b_{l}} \otimes I d_{S^{p}}\right)(y)\right\|_{L^{p}\left(\mathbb{R}, S^{p}\right)} \geqslant 2 c$. We deduce that there exists a continuous function $b: \mathbb{R} \rightarrow \mathbb{C}$ with compact support such that $\left\|C_{b}\right\|_{L^{p}(\mathbb{R}) \rightarrow L^{p}(\mathbb{R})} \leqslant 1$ and $\left\|C_{b}\right\|_{c b, L^{p}(\mathbb{R}) \rightarrow L^{p}(\mathbb{R})} \geqslant 2 c$. Thus there exists a continuous function $\mathbb{R} \stackrel{b}{\rightarrow} \mathbb{C}$ with compact support such that

$$
\left\|C_{b}\right\|_{L^{p}(\mathbb{R}) \rightarrow L^{p}(\mathbb{R})} \leqslant 1 \quad \text { and } \quad\left\|C_{b}\right\|_{c b, L^{p}(\mathbb{R}) \rightarrow L^{p}(\mathbb{R})} \geqslant 2 c .
$$

Now, we define the sequence $\left(a_{n}\right)_{n \geqslant 1}$ of complex sequences indexed by $\mathbb{Z}$ by, if $n \geqslant 1$ and $k \in \mathbb{Z}$

$$
a_{n, k}=\int_{0}^{1} \int_{0}^{1} \frac{1}{n} b\left(\frac{t-s+k}{n}\right) d s d t .
$$

Note that each sequence $a_{n}$ has only a finite number of non-zero term. Let $n \geqslant 1$. We introduce the conditional expectation $L^{p}(\mathbb{R}) \stackrel{\mathbb{E}_{n}}{\longrightarrow} L^{p}(\mathbb{R})$ with respect to the $\sigma$-algebra generated by the $\left[\frac{k}{n}, \frac{k+1}{n}[\right.$, $k \in \mathbb{Z}$. For every integer $n \geqslant 1$ and all $f \in L^{p}(\mathbb{R})$, we have

$$
\mathbb{E}_{n} f=n \sum_{k \in \mathbb{Z}}\left(\int_{\frac{k}{n}}^{\frac{k+1}{n}} f(t) d t\right) 1_{\left[\frac{k}{n}, \frac{k+1}{n}\right.}[
$$

(see [AbA, page 227]). Now, we define the linear map $\ell_{\mathbb{Z}}^{p} \stackrel{J_{n}}{\longrightarrow} \mathbb{E}_{n}\left(L^{p}(\mathbb{R})\right)$ by, if $u \in \ell_{\mathbb{Z}}^{p}$

$$
J_{n}(u)=n^{\frac{1}{p}} \sum_{k \in \mathbb{Z}} u_{k} 1_{\left[\frac{k}{n}, \frac{k+1}{n}\right.}[\cdot
$$

It is easy to check that the map $J_{n}$ is an isometry of $\ell_{\mathbb{Z}}^{p}$ onto the range $\mathbb{E}_{n}\left(L^{p}(\mathbb{R})\right)$ of $\mathbb{E}_{n}$. For any $u \in \ell_{\mathbb{Z}}^{p}$, we have

$$
\begin{aligned}
& \mathbb{E}_{n} C_{b} J_{n}(u)=n \sum_{k \in \mathbb{Z}}\left(\int_{\frac{k}{n}}^{\frac{k+1}{n}}\left(C_{b} J_{n}(u)\right)(t) d t\right) 1_{\left[\frac{k}{n}, \frac{k+1}{n}\right.}[ \\
& =n \sum_{k \in \mathbb{Z}}\left(\int_{\frac{k}{n}}^{\frac{k+1}{n}} \int_{-\infty}^{+\infty} b(t-s)\left(J_{n}(u)\right)(s) d s d t\right) 1_{\left[\frac{k}{n}, \frac{k+1}{n}\right.}[ \\
& =n \sum_{k \in \mathbb{Z}}\left(\int_{\frac{k}{n}}^{\frac{k+1}{n}} \int_{-\infty}^{+\infty} b(t-s) n^{\frac{1}{p}}\left(\sum_{j \in \mathbb{Z}} u_{j} 1_{\left[\frac{j}{n}, \frac{j+1}{n}\right.}[s)\right) d s d t\right) 1_{\left[\frac{k}{n}, \frac{k+1}{n}[\right.} \\
& =n^{1+\frac{1}{p}} \sum_{k \in \mathbb{Z}}\left(\sum_{j \in \mathbb{Z}} u_{j} \int_{\frac{k}{n}}^{\frac{k+1}{n}} \int_{\frac{j}{n}}^{\frac{j+1}{n}} b(t-s) d s d t\right) 1_{\left[\frac{k}{n}, \frac{k+1}{n}\right.}[ \\
& =n^{1+\frac{1}{p}} \sum_{k \in \mathbb{Z}}\left(\sum_{j \in \mathbb{Z}} u_{j} \int_{0}^{\frac{1}{n}} \int_{0}^{\frac{1}{n}} b\left(t-s+\frac{k-j}{n}\right) d s d t\right) 1_{\left[\frac{k}{n}, \frac{k+1}{n}[\right.} \\
& =n^{\frac{1}{p}} \sum_{k \in \mathbb{Z}}\left(\sum_{j \in \mathbb{Z}} u_{j} \int_{0}^{1} \int_{0}^{1} b\left(\frac{t-s+k-j}{n}\right) d s d t\right) 1_{\left[\frac{k}{n}, \frac{k+1}{n}[\right.}[ \\
& =n^{\frac{1}{p}} \sum_{k \in \mathbb{Z}}\left(\sum_{j \in \mathbb{Z}} u_{j} a_{n, k-j}\right) 1_{\left[\frac{k}{n}, \frac{k+1}{n}\right.}[ \\
& =J_{n} C_{a_{n}}(u)
\end{aligned}
$$


(where the equality (3.3) follows from the fact that the summation over $j \in \mathbb{Z}$ of (3.2) is finite). Thus we have the following commutative diagram

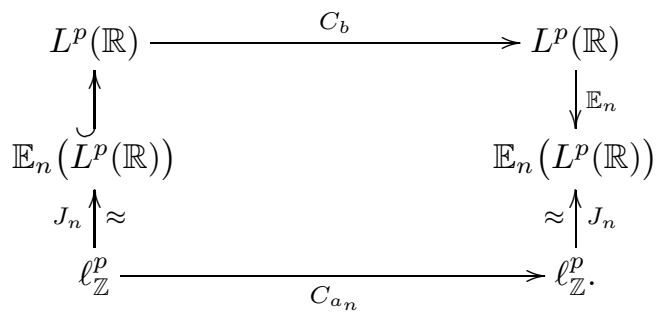

Then, for any integer $n \geqslant 1$, since $\left\|\mathbb{E}_{n}\right\|_{L^{p}(\mathbb{R}) \rightarrow L^{p}(\mathbb{R})} \leqslant 1$, we have the following estimate

$$
\left\|C_{a_{n}}\right\|_{\ell_{\mathbb{Z}}^{p} \rightarrow \ell_{\mathbb{Z}}^{p}} \leqslant\left\|C_{b}\right\|_{L^{p}(\mathbb{R}) \rightarrow L^{p}(\mathbb{R})} \leqslant 1 .
$$

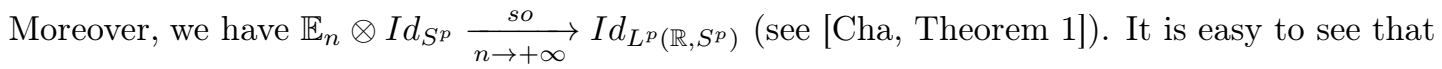

$$
\left(\mathbb{E}_{n} C_{b} \mathbb{E}_{n}\right) \otimes I d_{S^{p}} \underset{n \rightarrow+\infty}{\stackrel{s o}{\longrightarrow}} C_{b} \otimes I d_{S^{p}} .
$$

By the strong semicontinuity of the norm, we obtain that

$$
\left\|C_{b}\right\|_{c b, L^{p}(\mathbb{R}) \rightarrow L^{p}(\mathbb{R})} \leqslant \liminf _{n \rightarrow \infty}\left\|\mathbb{E}_{n} C_{b} \mathbb{E}_{n}\right\|_{c b, L^{p}(\mathbb{R}) \rightarrow L^{p}(\mathbb{R})} .
$$

Then, there exists an integer $n \geqslant 1$ such that

$$
\left\|C_{a_{n}}\right\|_{\ell_{\mathbb{Z}}^{p} \rightarrow \ell_{\mathbb{Z}}^{p}} \leqslant 1 \quad \text { and } \quad\left\|C_{a_{n}}\right\|_{c b, \ell_{\mathbb{Z}}^{p} \rightarrow \ell_{\mathbb{Z}}^{p}} \geqslant c .
$$

Thus, we prove the second assertion by shifting the obtained multiplier. Finally, we show the first assertion by the closed graph theorem, (2.3) for $X=\mathbb{C}$ and (2.4).

The paper Arh is a continuation of these investigations. The author proves that if $G$ is an arbitrary infinite locally compact abelian group, $1<p<\infty$ and $p \neq 2$ then there exists a bounded Fourier multiplier on $L^{p}(G)$ which is not completely bounded.

In the light of Corollary 2.3 and Theorem 3.5. it is natural to compare $\|P(\sigma)\|_{c b, S^{p} \rightarrow S^{p}}$ and $\|P(\sigma)\|_{S^{p} \rightarrow S^{p}}$. We finish the section by proving that these quantities are identical. It is a result due to É. Ricard. In order to prove it, we need the following notion of Schur multiplier. We equip $\mathbb{T}$ with its normalized Haar measure. We denote by $S^{p}\left(L^{2}(\mathbb{T})\right)$ the Schatten-von Neumann class associated with $B\left(L^{2}(\mathbb{T})\right)$. If $f \in L_{2}(\mathbb{T} \times \mathbb{T})$, we denote the associated Hilbert-Schmidt operator by

$$
\begin{aligned}
& K_{f}: L^{2}(\mathbb{T}) \quad \longrightarrow \quad L^{2}(\mathbb{T}) \\
& u \quad \longmapsto \int_{\mathbb{T}} u(z) f(z, \cdot) d z .
\end{aligned}
$$

A Schur multiplier on $S^{p}\left(L^{2}(\mathbb{T})\right)$ is a linear map $S^{p}\left(L^{2}(\mathbb{T})\right) \stackrel{T}{\rightarrow} S^{p}\left(L^{2}(\mathbb{T})\right)$ such that there exists a measurable function $\mathbb{T} \times \mathbb{T} \stackrel{\varphi}{\rightarrow} \mathbb{C}$ which satisfies, for any finite rank operator of the form $L^{2}(\mathbb{T}) \stackrel{K_{f}}{\longrightarrow}$ $L^{2}(\mathbb{T})$, the equality $T\left(K_{f}\right)=K_{\varphi f}$. We denote $T$ by $M_{\varphi}$ and we say that the function $\varphi$ is the symbol of the Schur multiplier $S^{p}\left(L^{2}(\mathbb{T})\right) \stackrel{M_{\varphi}}{\longrightarrow} S^{p}\left(L^{2}(\mathbb{T})\right.$ ) (see [BiS] and [LaS] for more details).

We denote by $L^{2}(\mathbb{T}) \stackrel{\mathcal{F}}{\rightarrow} \ell_{\mathbb{Z}}^{2}$ the Fourier transform. We define the isometry $\Psi$ by

$$
\begin{array}{rl}
\Psi: S^{p}\left(L^{2}(\mathbb{T})\right) & \longrightarrow \\
T & S_{\mathbb{Z}}^{p} \\
T & \mathcal{F} T \mathcal{F}^{-1} .
\end{array}
$$

Now, we can show the following proposition. 
Proposition 3.6 Suppose $1 \leqslant p \leqslant \infty$. For any complex polynomial $P$, we have

$$
\|P(\sigma)\|_{S^{p} \rightarrow S^{p}}=\|P(\sigma)\|_{c b, S^{p} \rightarrow S^{p}}\left(=\left\|P(\sigma) \otimes I d_{S^{p}}\right\|_{S^{p}\left(S^{p}\right) \rightarrow S^{p}\left(S^{p}\right)}\right) .
$$

Proof : It suffices to consider the case $1<p<\infty$. For any $n \in \mathbb{Z}$ and any finite rank operator of the form $K_{f}$, we have

$$
\begin{aligned}
\left(\Theta \Psi\left(K_{f}\right)\right)\left(e_{n}\right) & =S \mathcal{F} K_{f} \mathcal{F}^{-1} S^{-1}\left(e_{n}\right) \\
& =S \mathcal{F} K_{f}\left(z^{n-1}\right) \\
& =S \mathcal{F}\left(\int_{\mathbb{T}} z^{n-1} f(z, \cdot) d z\right) \\
& =S\left(\sum_{k \in \mathbb{Z}}\left(\int_{\mathbb{T}^{2}} z^{n}{\overline{z^{\prime}}}^{k} f\left(z, z^{\prime}\right) d z d z^{\prime}\right) e_{k}\right) \\
& =\sum_{k \in \mathbb{Z}}\left(\int_{\mathbb{T}^{2}} z^{n-1}{\overline{z^{\prime}}}^{k} f\left(z, z^{\prime}\right) d z d z^{\prime}\right) e_{k+1} .
\end{aligned}
$$

Now we define the function $\mathbb{T} \times \mathbb{T} \stackrel{\varphi}{\rightarrow} \mathbb{C}$ by $\varphi\left(z, z^{\prime}\right)=z^{-1} z^{\prime}$ where $z, z^{\prime} \in \mathbb{T}$. Then, for any $n \in \mathbb{Z}$ and any finite rank operator of the form $K_{f}$, we have

$$
\begin{aligned}
\left(\Psi M_{\varphi}\left(K_{f}\right)\right)\left(e_{n}\right) & =\mathcal{F} K_{\varphi f} \mathcal{F}^{-1}\left(e_{n}\right) \\
& =\mathcal{F}\left(\int_{\mathbb{T}} z^{n} \varphi(z, \cdot) f(z, \cdot) d z\right) \\
& =\sum_{k \in \mathbb{Z}}\left(\int_{\mathbb{T}^{2}} z^{n}{\overline{z^{\prime}}}^{k} \varphi\left(z, z^{\prime}\right) f\left(z, z^{\prime}\right) d z d z^{\prime}\right) e_{k} \\
& =\sum_{k \in \mathbb{Z}}\left(\int_{\mathbb{T}^{2}} z^{n-1}{\overline{z^{\prime}}}^{k-1} f\left(z, z^{\prime}\right) d z d z^{\prime}\right) e_{k} \\
& =\sum_{k \in \mathbb{Z}}\left(\int_{\mathbb{T}^{2}} z^{n-1}{\overline{z^{\prime}}}^{k} f\left(z, z^{\prime}\right) d z d z^{\prime}\right) e_{k+1} .
\end{aligned}
$$

Then, for any complex polynomial $P$, we have the following commutative diagram

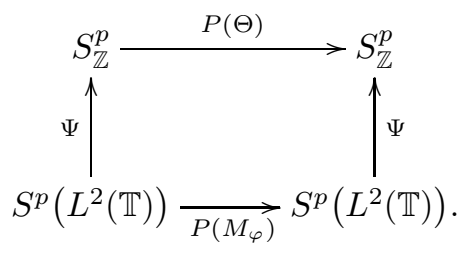

Furthermore, for any complex polynomial $P$, we have $P\left(M_{\varphi}\right)=M_{P(\varphi)}$. Moreover, the Schur multiplier $S^{p}\left(L^{2}(\mathbb{T})\right) \stackrel{M_{P(\varphi)}}{\longrightarrow} S^{p}\left(L^{2}(\mathbb{T})\right)$ has a continuous symbol whose the support has no isolated point. By [LaS, Theorem 1.19], we deduce that the norm and the completely bounded norm of $P\left(M_{\varphi}\right)$ coincide. Since $\Psi$ is a complete isometry, we obtain the result by (2.5). 


\section{Positive results}

Let $M$ and $N$ be von Neumann algebras equipped with faithful semifinite normal traces $\tau_{M}$ and $\tau_{N}$. Let $M \stackrel{T}{\rightarrow} N$ a positive linear map. We say that $T$ is trace preserving if for all $x \in L^{1}(M) \cap M_{+}$we have $\tau_{N}(T(x))=\tau_{M}(x)$. We will use the following straightforward extension of [JuX, Lemma 1.1].

Lemma 4.1 Let $M$ and $N$ be von Neumann algebras equipped with faithful semifinite normal traces. Let $M \stackrel{T}{\rightarrow} N$ be a trace preserving unital normal positive map. Suppose $1 \leqslant p<\infty$. Then $T$ induces a contraction $L^{p}(M) \stackrel{T}{\rightarrow} L^{p}(N)$. Moreover, if $M \stackrel{T}{\rightarrow} N$ is an one-to-one normal unital $*$-homomorphism, $T$ induces an isometry $L^{p}(M) \stackrel{T}{\rightarrow} L^{p}(N)$.

Let $M$ be a von Neumann algebra equipped with faithful semifinite normal trace $\tau$ and $N$ a von Neumann subalgebra such that the restriction of $\tau$ is still semifinite. Then, it is well-known that the extension $L^{p}(M) \stackrel{\mathbb{E}}{\rightarrow} L^{p}(N)$ of the canonical faithful normal trace preserving conditional $M \stackrel{\mathbb{E}}{\rightarrow} N$ is a contractive projection.

Consider the situation where $M \stackrel{T}{\rightarrow} M$ is a linear map such there exists a von Neumann algebra $N$ equipped with a faithful semifinite normal trace, a unital trace preserving $*$-automorphism $N \stackrel{U}{\rightarrow} N$, a unital normal trace preserving one-to-one $*$-homomorphism $M \stackrel{J}{\rightarrow} N$ such that,

$$
T^{k}=\mathbb{E} U^{k} J
$$

for any integer $k \geqslant 0$, where $N \stackrel{\mathbb{E}}{\rightarrow} M$ is the canonical faithful normal trace preserving conditional expectation associated with $J$. Then, for all $1 \leqslant p<\infty$, the maps $N \stackrel{U}{\rightarrow} N$ and $M \stackrel{J}{\rightarrow} N$ extend to isometries $L_{p}(N) \stackrel{U}{\rightarrow} L_{p}(N)$ and $L^{p}(M) \stackrel{J}{\rightarrow} L^{p}(N)$ and the map $N \stackrel{\mathbb{E}}{\rightarrow} M$ extends to a contractive projection $L^{p}(N) \stackrel{\mathbb{E}}{\rightarrow} L^{p}(M)$ such that (4.1) is also true for the induced map $L^{p}(M) \stackrel{T}{\rightarrow} L^{p}(M)$.

In order to prove Theorems 1.5 and 1.6, we need to use fermion algebras. Since we will study maps between $q$-deformed algebras, we recall directly several facts about these more general algebras in the context of [BKS]. We denote by $S_{n}$ the symmetric group. If $\sigma$ is a permutation of $S_{n}$ we denote by $|\sigma|$ the number $\operatorname{card}\{(i, j) \mid 1 \leqslant i, j \leqslant n, \sigma(i)>\sigma(j)\}$ of inversions of $\sigma$. Let $H$ be a real Hilbert space with complexification $H_{\mathbb{C}}$. If $-1 \leqslant q<1$ the $q$-Fock space over $H$ is

$$
\mathcal{F}_{q}(H)=\mathbb{C} \Omega \oplus \bigoplus_{n \geqslant 1} H_{\mathbb{C}}^{\otimes_{n}}
$$

where $\Omega$ is a unit vector, called the vacuum and where the scalar product on $H_{\mathbb{C}}^{\otimes_{n}}$ is given by

$$
\left\langle h_{1} \otimes \cdots \otimes h_{n}, k_{1} \otimes \cdots \otimes k_{n}\right\rangle_{q}=\sum_{\sigma \in S_{n}} q^{|\sigma|}\left\langle h_{1}, k_{\sigma(1)}\right\rangle_{H_{\mathbb{C}}} \cdots\left\langle h_{n}, k_{\sigma(n)}\right\rangle_{H_{\mathbb{C}}} .
$$

If $q=-1$, we must first divide out by the null space, and we obtain the usual antisymmetric Fock space. The creation operator $l(e)$ for $e \in H$ is given by

$$
l(e): \begin{array}{ccc}
\mathcal{F}_{q}(H) & \longrightarrow & \mathcal{F}_{q}(H) \\
h_{1} \otimes \cdots \otimes h_{n} & \longmapsto & e \otimes h_{1} \otimes \cdots \otimes h_{n} .
\end{array}
$$

They satisfy the $q$-relation

$$
l(f)^{*} l(e)-q l(e) l(f)^{*}=\langle f, e\rangle_{H} I d_{\mathcal{F}_{q}(H)}
$$


We denote by $\mathcal{F}_{q}(H) \stackrel{\omega(e)}{\longrightarrow} \mathcal{F}_{q}(H)$ the selfadjoint operator $l(e)+l(e)^{*}$. The $q$-von Neumann algebra $\Gamma_{q}(H)$ is the von Neumann algebra generated by the operators $\omega(e)$ where $e \in H$. It is a finite von Neumann algebra with the trace $\tau$ defined by $\tau(x)=\langle\Omega, x . \Omega\rangle_{\mathcal{F}_{q}(H)}$ where $x \in \Gamma_{q}(H)$.

Let $H$ and $K$ be real Hilbert spaces and $H \stackrel{T}{\rightarrow} K$ be a contraction with complexification $H_{\mathbb{C}} \stackrel{T_{\mathbb{C}}}{\longrightarrow} K_{\mathbb{C}}$. We define the following linear map

$$
\begin{array}{cccc}
\mathcal{F}_{q}(T): & \mathcal{F}_{q}(H) & \longrightarrow & \mathcal{F}_{q}(K) \\
h_{1} \otimes \cdots \otimes h_{n} & \longmapsto & T_{\mathbb{C}} h_{1} \otimes \cdots \otimes T_{\mathbb{C}} h_{n} .
\end{array}
$$

Then there exists a unique map $\Gamma_{q}(H) \stackrel{\Gamma_{q}(T)}{\longrightarrow} \Gamma_{q}(H)$ such that for every $x \in \Gamma_{q}(H)$ we have

$$
\left(\Gamma_{q}(T)(x)\right) \Omega=\mathcal{F}_{q}(T)(x \Omega) .
$$

This map is normal, unital, completely positive and trace preserving. If $H \stackrel{T}{\rightarrow} K$ is an isometry, $\Gamma_{q}(T)$ is an injective $*$-homomorphism. If $1 \leqslant p<\infty$, it extends to a contraction $L^{p}\left(\Gamma_{q}(H)\right) \stackrel{\Gamma_{q}(T)}{\longrightarrow}$ $L^{p}\left(\Gamma_{q}(K)\right)$.

We are mainly concerned with the fermion algebra $\Gamma_{-1}(H)$. In this case, recall that if $e \in H$ has norm 1, then the operator $\omega(e)$ satisfies $\omega(e)^{2}=I d_{\mathcal{F}_{-1}(H)}$. Moreover, we need the following Wick formula, (see [Boz, page 2] and [EfP, Corollary 2.1]). In order to state this, we denote, if $k \geqslant 1$ is an integer, by $\mathcal{P}_{2}(2 k)$ the set of 2 -partitions of the set $\{1,2, \ldots, 2 k\}$. If $\mathcal{V} \in \mathcal{P}_{2}(2 k)$ we let $c(\mathcal{V})$ the number of crossings of $\mathcal{V}$, which is given, by the number of pairs of blocks of $\mathcal{V}$ which cross (see [EfP] page 8630] for a precise definition). Then, if $f_{1}, \ldots, f_{2 k} \in H$ we have

$$
\tau\left(\omega\left(f_{1}\right) \omega\left(f_{2}\right) \cdots \omega\left(f_{2 k}\right)\right)=\sum_{\mathcal{V} \in \mathcal{P}_{2}(2 k)}(-1)^{c(\mathcal{V})} \prod_{(i, j) \in \mathcal{V}}\left\langle f_{i}, f_{j}\right\rangle_{H} .
$$

In particular, for all $e, f \in H$, we have

$$
\tau(\omega(e) \omega(f))=\langle e, f\rangle_{H}
$$

Let $A=\left[a_{i j}\right]_{i, j \in I}$ be a matrix of $\mathbb{M}_{I}$. By definition, the Schur multiplier on $B\left(\ell_{I}^{2}\right)$ associated with this matrix is the unbounded linear operator $M_{A}$ whose domain is the space of all $B=\left[b_{i j}\right]_{i, j \in I}$ of $B\left(\ell_{I}^{2}\right)$ such that $\left[a_{i j} b_{i j}\right]_{i, j \in I}$ belongs to $B\left(\ell_{I}^{2}\right)$, and whose action on $B=\left[b_{i j}\right]_{i, j \in I}$ is given by $M_{A}(B)=\left[a_{i j} b_{i j}\right]_{i, j \in I}$. For all $i, j \in I$, the matrix $e_{i j}$ belongs to $D\left(M_{A}\right)$, hence $M_{A}$ is densely defined for the weak* topology. Suppose $1 \leqslant p<\infty$. If for any $B \in S_{I}^{p}$, we have $B \in D\left(M_{A}\right)$ and the matrix $M_{A}(B)$ represents an element of $S_{I}^{p}$, by the closed graph theorem, the matrix $A$ of $\mathbb{M}_{I}$ defines a bounded Schur multiplier $S_{I}^{p} \stackrel{M_{A}}{\longrightarrow} S_{I}^{p}$. We have a similar statement for bounded Schur multipliers on $B\left(\ell_{I}^{2}\right)$.

Recall that a matrix $A$ of $\mathbb{M}_{I}$ defines a contractive Schur multiplier $B\left(\ell_{I}^{2}\right) \stackrel{M_{A}}{\longrightarrow} B\left(\ell_{I}^{2}\right)$ if and only if there exists an index set $K$ and norm 1 vectors $h_{i} \in \ell_{K}^{2}$ and $k_{j} \in \ell_{K}^{2}$ such that for all $i, j \in I$ we have $a_{i, j}=\left\langle h_{i}, k_{j}\right\rangle_{\ell_{K}^{2}}($ see $[\overline{\mathrm{Pau}}])$. If all entries of $A$ are real numbers, we can take the real vector space $\ell_{K}^{2}(\mathbb{R})$ instead of the complex vector space $\ell_{K}^{2}$. Finally, recall that every contractive Schur multiplier $B\left(\ell_{I}^{2}\right) \stackrel{M_{A}}{\longrightarrow} B\left(\ell_{I}^{2}\right)$ is completely contractive (see [Pau]).

We say that a matrix $A$ of $\mathbb{M}_{I}$ induces a completely positive Schur multiplier $B\left(\ell_{I}^{2}\right) \stackrel{M_{A}}{\longrightarrow} B\left(\ell_{I}^{2}\right)$ if and only if for any finite set $F \subset I$ the matrix $\left[a_{i, j}\right]_{i, j \in F}$ is positive (see $[\mathrm{Pau}]$ ). An other well-known characterization is that there exists vectors $h_{i} \in \ell_{K}^{2}(\mathbb{C})$ of norm 1 such that for all $i, j \in I$ we have $a_{i, j}=\left\langle h_{i}, h_{j}\right\rangle_{\ell_{K}^{2}}$. If $A$ is a real matrix, we can use the real vector space $\ell_{K}^{2}(\mathbb{R})$ instead of the complex vector space $\ell_{K}^{2}$. 
Let $M$ be a von Neumann algebra equipped with a semifinite normal faithful trace $\tau$. Suppose that $M \stackrel{T}{\rightarrow} M$ is a normal contraction. We say that $T$ is selfadjoint if for all $x, y \in M \cap L^{1}(M)$ we have

$$
\tau\left(T(x) y^{*}\right)=\tau\left(x(T y)^{*}\right)
$$

In this case, it is easy to see that the restriction $T \mid M \cap L^{1}(M)$ extends to a contraction $L^{1}(M) \stackrel{T}{\rightarrow}$ $L^{1}(M)$. By complex interpolation, for any $1 \leqslant p \leqslant \infty$, we obtain a contractive map $L^{p}(M) \stackrel{T}{\rightarrow} L^{p}(M)$. Moreover, the operator $L^{2}(M) \stackrel{T}{\rightarrow} L^{2}(M)$ is selfadjoint. If $M \stackrel{T}{\rightarrow} M$ is a normal selfadjoint complete contraction, it is easy to see that the map $L^{p}(M) \stackrel{T}{\rightarrow} L^{p}(M)$ is completely contractive for all $1 \leqslant p \leqslant$ $\infty$. It is easy to see that a contractive Schur multiplier $B\left(\ell_{I}^{2}\right) \stackrel{M_{A}}{\longrightarrow} B\left(\ell_{I}^{2}\right)$ associated with a matrix $A$ of $\mathbb{M}_{I}$ is selfadjoint if and only if all entries of $A$ are real.

In order to prove the next theorem, we need the following notion of infinite tensor product of von Neumann algebras, see [Tak3]. Given a sequence $\left(M_{n}, \tau_{n}\right)_{n \in \mathbb{Z}}$ of von Neumann algebras $M_{n}$ equipped with faithful normal finite traces $\tau_{n}$, then on the infinite minimal $C^{*}$-tensor product of the algebras $\left(M_{n}\right)_{n \in \mathbb{Z}}$ there is a well-defined infinite product state $\cdots \otimes \tau_{-1} \otimes \tau_{0} \otimes \tau_{1} \otimes \cdots$. The weak operator closure of the GNS-representation of the infinite $C^{*}$-tensor product of $\left(M_{n}\right)_{n \in \mathbb{Z}}$ with respect to the state $\cdots \otimes \tau_{-1} \otimes \tau_{0} \otimes \tau_{1} \otimes \cdots$ yields a von Neumann algebra, called the infinite tensor product of von Neumann algebras $M_{n}$ with respect to the traces $\tau_{n}$. We will denote this algebra by $\bar{\bigotimes}_{n \in \mathbb{Z}}\left(M_{n}, \tau_{n}\right)$. The state $\cdots \otimes \tau_{-1} \otimes \tau_{0} \otimes \tau_{1} \otimes \cdots$ extends to a faithful normal finite trace on $\bar{\bigotimes}_{n \in \mathbb{Z}}\left(M_{n}, \tau_{n}\right)$ which we still denote by $\cdots \otimes \tau_{-1} \otimes \tau_{0} \otimes \tau_{1} \otimes \cdots$.

The following theorem states that we can dilate some Schur multipliers. The construction (and the one of Theorem 4.6] is inspired by the work of É. Ricard [Ric].

Theorem 4.2 Let $B\left(\ell_{I}^{2}\right) \stackrel{M_{A}}{\longrightarrow} B\left(\ell_{I}^{2}\right)$ be a unital completely positive Schur multiplier associated with a real-valued matrix $A$. Then there exists a hyperfinite von Neumann algebra $M$ equipped with a semifinite normal faithful trace, a unital trace preserving *-automorphism $M \stackrel{U}{\rightarrow} M$, a unital trace preserving one-to-one normal *-homomorphism $B\left(\ell_{I}^{2}\right) \stackrel{J}{\rightarrow} M$ such that

$$
\left(M_{A}\right)^{k}=\mathbb{E} U^{k} J
$$

for any integer $k \geqslant 0$, where $M \stackrel{\mathbb{E}}{\rightarrow} B\left(\ell_{I}^{2}\right)$ is the canonical faithful normal trace preserving conditional expectation associated with $J$.

Proof : Since the map $B\left(\ell_{I}^{2}\right) \stackrel{M_{A}}{\longrightarrow} B\left(\ell_{I}^{2}\right)$ is completely positive we can define a positive symmetric bilinear form $\langle\cdot, \cdot\rangle_{\ell^{2, A}}$ on the real span of the $e_{i}$, where $i \in I$, by:

$$
\left\langle e_{i}, e_{j}\right\rangle_{\ell^{2, A}}=a_{i j} .
$$

We denote by $\ell^{2, A}$ the completion of the real pre-Hilbert obtained by quotient by the corresponding kernel. For all $i$ of $I$ we still denote by $e_{i}$ the class of $e_{i}$ in $\ell^{2, A}$. Now we define the von Neumann algebra $M$ by

$$
M=B\left(\ell_{I}^{2}\right) \bar{\otimes}\left(\overline{\bigotimes_{n \in \mathbb{Z}}}\left(\Gamma_{-1}\left(\ell^{2, A}\right), \tau\right)\right) .
$$

Since the von Neumann algebra $\Gamma_{-1}\left(\ell^{2, A}\right)$ is hyperfinite, the von Neumann algebra $M$ is also hyperfinite. We define the element $d$ of $M$ by

$$
d=\sum_{i \in I} e_{i i} \otimes \cdots \otimes I \otimes \omega\left(e_{i}\right) \otimes I \otimes \cdots
$$


where $\omega\left(e_{i}\right)$ is in position 0 . Recall that $M_{A}$ is unital. Then it is not difficult to see that $d$ is a symmetry, i.e. a selfadjoint unitary element. We equip the von Neumann algebra $M$ with the faithful semifinite normal trace $\tau_{M}=\operatorname{Tr} \otimes \cdots \otimes \tau \otimes \tau \otimes \cdots$. We denote by $M \stackrel{\mathbb{E}}{\rightarrow} B\left(\ell_{I}^{2}\right)$ the canonical faithful normal trace preserving conditional expectation of $M$ onto $B\left(\ell_{I}^{2}\right)$. We have

$$
\mathbb{E}=I d_{B\left(\ell_{I}^{2}\right)} \otimes \cdots \otimes \tau \otimes \tau \otimes \cdots
$$

We define the canonical injective normal unital $*$-homomorphism

$$
\begin{array}{ccc}
J: B\left(\ell_{I}^{2}\right) & \longrightarrow \\
x & \longmapsto x \otimes \cdots \otimes I \otimes I \otimes \cdots .
\end{array}
$$

Clearly, $J$ preserves the traces. We define the right shift

$$
\begin{aligned}
& \mathscr{S}: \bar{\bigotimes}_{n \in \mathbb{Z}}\left(\Gamma_{-1}\left(\ell^{2, A}\right), \tau\right) \longrightarrow \bar{\bigotimes}_{n \in \mathbb{Z}}\left(\Gamma_{-1}\left(\ell^{2, A}\right), \tau\right) \\
& \cdots \otimes x_{0} \otimes x_{1} \otimes \cdots \quad \longmapsto \quad \cdots \otimes x_{-1} \otimes x_{0} \otimes \cdots \text {. }
\end{aligned}
$$

Now, we define the linear map

$$
\begin{array}{ccc}
U: M & \longrightarrow & M \\
y & \longmapsto d\left(\left(I d_{B\left(\ell_{I}^{2}\right)} \otimes \mathscr{S}\right)(y)\right) d .
\end{array}
$$

The map $M \stackrel{U}{\rightarrow} M$ is a unital *-automorphism of $M$. Moreover, it is easy to see that $M \stackrel{U}{\rightarrow} M$ preserves the trace $\tau_{M}$. Now, we will show that, for any positive integer $k$, we have, for all $x \in B\left(\ell_{I}^{2}\right)$

$$
U^{k} \circ J(x)=\sum_{i, j \in I} x_{i j} e_{i j} \otimes \cdots \otimes I \otimes \underbrace{\omega\left(e_{i}\right) \omega\left(e_{j}\right) \otimes \cdots \otimes \omega\left(e_{i}\right) \omega\left(e_{j}\right)}_{k \text { factors }} \otimes I \otimes \cdots
$$

by induction on $k$, where the first $\omega\left(e_{i}\right) \omega\left(e_{j}\right)$ is in position 0 . The statement clearly holds for $k=0$. Now, assume (4.5). For all $x \in B\left(\ell_{I}^{2}\right)$, we have

$$
\begin{aligned}
& U^{k+1} \circ J(x)=d\left(\left(I d_{B\left(\ell_{I}^{2}\right)} \otimes \mathscr{S}\right)\left(U^{k} \circ J(x)\right)\right) d \\
&=d\left(\left(I d_{B\left(\ell_{I}^{2}\right)} \otimes \mathscr{S}\right)\left(\sum_{i, j \in I} x_{i j} e_{i j} \otimes \cdots \otimes I \otimes \omega\left(e_{i}\right) \omega\left(e_{j}\right) \otimes \cdots \otimes \omega\left(e_{i}\right) \omega\left(e_{j}\right) \otimes I \otimes \cdots\right)\right) d \\
&=\left(\sum_{r \in I} e_{r r} \otimes \cdots \otimes I \otimes \omega\left(e_{r}\right) \otimes I \otimes \cdots\right)\left(\sum_{i, j \in I} x_{i j} e_{i j} \otimes \cdots \otimes I \otimes I \otimes \omega\left(e_{i}\right) \omega\left(e_{j}\right) \otimes \cdots\right. \\
&=\sum_{i, j, r, s \in I} x_{i j} e_{r r} e_{i j} e_{s s} \otimes \cdots \otimes I \otimes \omega\left(e_{r}\right) \omega\left(e_{s}\right) \otimes \omega\left(e_{i}\right) \omega\left(e_{j}\right) \otimes \cdots \otimes \omega\left(e_{i}\right) \omega\left(e_{j}\right) \otimes I \otimes \cdots \\
&= \sum_{i, j \in I} x_{i j} e_{i j} \otimes \cdots \otimes I \otimes \omega\left(e_{i}\right) \omega\left(e_{j}\right) \otimes \omega\left(e_{i}\right) \omega\left(e_{j}\right) \otimes \cdots \otimes \omega\left(e_{i}\right) \omega\left(e_{j}\right) \otimes I \otimes \cdots
\end{aligned}
$$


We obtained the statement (4.5) for $k+1$. Then, we deduce that for any positive integer $k$ and any $x \in B\left(\ell_{I}^{2}\right)$ we have

$$
\begin{aligned}
\mathbb{E} & \circ U^{k} \circ J(x) \\
& =\left(I d_{S_{I}^{p}} \otimes \cdots \otimes \tau \otimes \cdots\right)\left(\sum_{i, j \in I} x_{i j} e_{i j} \otimes \cdots \otimes I \otimes \omega\left(e_{i}\right) \omega\left(e_{j}\right) \otimes \cdots \otimes \omega\left(e_{i}\right) \omega\left(e_{j}\right) \otimes I \otimes \cdots\right) \\
& =\sum_{i, j \in I} \tau\left(\omega\left(e_{i}\right) \omega\left(e_{j}\right)\right)^{k} x_{i j} e_{i j} \\
& =\sum_{i, j \in I}\left(\left\langle e_{i}, e_{j}\right\rangle_{\ell^{2}, A}\right)^{k} x_{i j} e_{i j} \quad \text { by (4.3) } \\
& =\sum_{i, j \in I}\left(a_{i j}\right)^{k} x_{i j} e_{i j} \quad \text { by (4.4) } \\
& =\left(M_{A}\right)^{k}(x) .
\end{aligned}
$$

Thus, for any positive integer $k$, we have

$$
\left(M_{A}\right)^{k}=\mathbb{E} \circ U^{k} \circ J
$$

The proof is complete.

Corollary 4.3 Let $B\left(\ell_{I}^{2}\right) \stackrel{M_{A}}{\longrightarrow} B\left(\ell_{I}^{2}\right)$ be a contractive Schur multiplier associated with a real-valued matrix A. Suppose $1<p<\infty$. Then, the induced Schur multiplier $S_{I}^{p} \stackrel{M_{A}}{\longrightarrow} S_{I}^{p}$ satisfies the noncommutative Matsaev's property. More precisely, for any complex polynomial $P$, we have

$$
\left\|P\left(M_{A}\right)\right\|_{c b, S_{I}^{p} \rightarrow S_{I}^{p}} \leqslant\|P\|_{p, S^{p}}
$$

Proof : Suppose that $B\left(\ell_{I}^{2}\right) \stackrel{M_{A}}{\longrightarrow} B\left(\ell_{I}^{2}\right)$ is a contractive Schur multiplier associated with a real matrix $A$ of $\mathbb{M}_{I}$. There exists a set $K$ and norm 1 vectors $h_{i} \in \ell_{K}^{2}(\mathbb{R})$ and $k_{i} \in \ell_{K}^{2}(\mathbb{R})$ such that for all $i, j \in I$ we have $a_{i, j}=\left\langle h_{i}, k_{j}\right\rangle_{\ell_{K}^{2}(\mathbb{R})}$. Now we define the following matrices of $\mathbb{M}_{I}$

$$
B=\left[\left\langle h_{i}, h_{j}\right\rangle_{\ell_{K}^{2}(\mathbb{R})}\right]_{i, j \in I}, \quad C=\left[\left\langle k_{i}, k_{j}\right\rangle_{\ell_{K}^{2}(\mathbb{R})}\right]_{i, j \in I} \text { and } D=\left[\left\langle k_{i}, h_{j}\right\rangle_{\ell_{K}^{2}(\mathbb{R})}\right]_{i, j \in I} .
$$

For all $i \in I$ and all $n \in\{1,2\}$, we define the norm 1 vector $l_{(n, i)}$ of $\ell_{K}^{2}(\mathbb{R})$ by

$$
l_{(n, i)}=\left\{\begin{array}{cl}
h_{i} & \text { if } n=1 \text { and } i \in I \\
k_{i} & \text { if } n=2 \text { and } i \in I .
\end{array}\right.
$$

Now, by the identification $\mathbb{M}_{2}\left(\mathbb{M}_{I}\right) \simeq \mathbb{M}_{\{1,2\} \times I}$, the matrix $\left[\begin{array}{cc}B & A \\ D & C\end{array}\right]$ of $\mathbb{M}_{2}\left(\mathbb{M}_{I}\right)$ identifies to the matrix

$$
F=\left[\left\langle l_{n, i}, l_{m, j}\right\rangle_{\ell_{K}^{2}(\mathbb{R})}\right]_{(n, i) \in\{1,2\} \times I,(m, j) \in\{1,2\} \times I}
$$

of $\mathbb{M}_{\{1,2\} \times I}$. The Schur multiplier $B\left(\ell_{\{1,2\} \times I}^{2}\right) \stackrel{M_{F}}{\longrightarrow} B\left(\ell_{\{1,2\} \times I}^{2}\right)$ associated with this matrix is unital and completely positive. Moreover, since the matrix $F$ is real, $B\left(\ell_{\{1,2\} \times I}^{2}\right) \stackrel{M_{F}}{\longrightarrow} B\left(\ell_{\{1,2\} \times I}^{2}\right)$ is 
selfadjoint. Let $1<p<\infty$. For any complex polynomial $P$, we have

$$
\begin{aligned}
\left\|P\left(M_{A}\right)\right\|_{S_{I}^{p} \rightarrow S_{I}^{p}} & \leqslant\left\|\left[\begin{array}{ll}
P\left(M_{B}\right) & P\left(M_{A}\right) \\
P\left(M_{D}\right) & P\left(M_{C}\right)
\end{array}\right]\right\|_{S_{2}^{p}\left(S_{I}^{p}\right) \rightarrow S_{2}^{p}\left(S_{I}^{p}\right)} \\
& =\left\|P\left(M_{F}\right)\right\|_{S_{\{1,2\} \times I}^{p} \rightarrow S_{\{1,2\} \times I}^{p}}
\end{aligned}
$$

Now, according to Theorem 4.2 remarks following Lemma 4.1 and Corollary 2.6, the Schur multiplier $M_{F}$ satisfies the noncommutative Matsaev's property. We deduce that $M_{A}$ also satisfies this property. Moreover, in applying this result to the Schur multiplier $M_{I \otimes A}\left(=I \otimes M_{A}\right)$, we obtain the inequality for the completely bounded norm.

We pass to Fourier multipliers on discrete groups. Suppose that $G$ is a discrete group. We denote by $e_{G}$ the neutral element of $G$. We denote by $\ell_{G}^{2} \stackrel{\lambda(g)}{\longrightarrow} \ell_{G}^{2}$ the unitary operator of left translation by $g$ and $\operatorname{VN}(G)$ the von Neumann algebra of $G$ spanned by the $\lambda(g)$ where $g \in G$. It is an finite algebra with trace given by

$$
\tau_{G}(x)=\left\langle\varepsilon_{e_{G}}, x\left(\varepsilon_{e_{G}}\right)\right\rangle_{\ell_{G}^{2}}
$$

where $\left(\varepsilon_{g}\right)_{g \in G}$ is the canonical basis of $\ell_{G}^{2}$ and $x \in \mathrm{VN}(G)$. A Fourier multiplier is a normal linear map $\mathrm{VN}(G) \stackrel{T}{\rightarrow} \mathrm{VN}(G)$ such that there exists a function $G \stackrel{t}{\rightarrow} \mathbb{C}$ such that for all $g \in G$ we have $T\left((\lambda(g))=t_{g} \lambda(g)\right.$. In this case, we denote $T$ by

$$
\begin{aligned}
M_{t}: \mathrm{VN}(G) & \longrightarrow \mathrm{VN}(G) \\
\lambda(g) & \longmapsto t_{g} \lambda(g) .
\end{aligned}
$$

It is easy to see that a contractive Fourier multiplier $\mathrm{VN}(G) \stackrel{M_{t}}{\longrightarrow} \mathrm{VN}(G)$ is selfadjoint if and only if $G \stackrel{t}{\rightarrow} \mathbb{C}$ is a real function. It is well-known that a Fourier multiplier $\mathrm{VN}(G) \stackrel{M_{t}}{\rightarrow} \mathrm{VN}(G)$ is completely positive if and only if the function $t$ is positive definite. If the discrete group $G$ is amenable, by [DCH], Corollary 1.8], every contractive Fourier multiplier $\mathrm{VN}(G) \stackrel{M_{t}}{\longrightarrow} \mathrm{VN}(G)$ is completely contractive. Recall the following particular case of the transfer result [NeR, Theorem 2.7].

Theorem 4.4 Let $G$ be an amenable discrete group. Suppose $1<p<\infty$. Let $G \stackrel{t}{\rightarrow} \mathbb{R}$ a function. Let $A$ be the matrix of $\mathbb{M}_{G}$ defined by $a_{g, h}=t_{g h^{-1}}$ where $g, h \in G$. The Fourier multiplier $M_{t}$ is completely bounded on $L^{p}(\mathrm{VN}(G))$ if and only if the Schur multiplier $M_{A}$ is completely bounded on $S_{G}^{p}$. In this case, we have

$$
\left\|M_{t}\right\|_{c b, L^{p}(\mathrm{VN}(G)) \rightarrow L^{p}(\mathrm{VN}(G))}=\left\|M_{A}\right\|_{c b, S_{G}^{p} \rightarrow S_{G}^{p}}
$$

Now, we can prove the next result.

Corollary 4.5 Let $G$ be an amenable discrete group. Let $\mathrm{VN}(G) \stackrel{M_{t}}{\longrightarrow} \mathrm{VN}(G)$ be a contractive Fourier multiplier associated with a real function $G \stackrel{t}{\rightarrow} \mathbb{R}$. Suppose $1<p<\infty$. Then, the induced Fourier multiplier $L^{p}(\mathrm{VN}(G)) \stackrel{M_{t}}{\longrightarrow} L^{p}(\mathrm{VN}(G))$ satisfies the noncommutative Matsaev's property. More precisely, for any complex polynomial $P$, we have

$$
\left\|P\left(M_{t}\right)\right\|_{c b, L^{p}(\mathrm{VN}(G)) \rightarrow L^{p}(\mathrm{VN}(G))} \leqslant\|P\|_{p, S^{p}} .
$$

Proof : We define the matrix $A$ of $\mathbb{M}_{G}$ by $a_{g, h}=t_{g h^{-1}}$ where $g, h \in G$. By (4.6), for any complex polynomial $P$ and all $1<p<\infty$, we have

$$
\begin{aligned}
\left\|P\left(M_{t}\right)\right\|_{c b, L^{p}(\mathrm{VN}(G)) \rightarrow L^{p}(\mathrm{VN}(G))} & =\left\|P\left(M_{A}\right)\right\|_{c b, S_{G}^{p} \rightarrow S_{G}^{p}} \\
& =\left\|P\left(I d_{S^{p}} \otimes M_{A}\right)\right\|_{S^{p}\left(S_{G}^{p}\right) \rightarrow S^{p}\left(S_{G}^{p}\right)} \\
& =\left\|P\left(M_{I \otimes A}\right)\right\|_{S^{p}\left(S_{G}^{p}\right) \rightarrow S^{p}\left(S_{G}^{p}\right)}
\end{aligned}
$$


Since $G$ is amenable, the Fourier multiplier $\operatorname{VN}(G) \stackrel{M_{t}}{\longrightarrow} \mathrm{VN}(G)$ is completely contractive. Moreover, the map $\operatorname{VN}(G) \stackrel{M_{t}}{\longrightarrow} \operatorname{VN}(G)$ is selfadjoint. Thus, for any $1<p<\infty$, the map $L^{p}(\operatorname{VN}(G)) \stackrel{M_{t}}{\longrightarrow}$ $L^{p}(\operatorname{VN}(G))$ is completely contractive. Then, by (4.6), for any $1<p<\infty$, we have

$$
\begin{aligned}
\left\|M_{I \otimes A}\right\|_{S^{p}\left(S_{G}^{p}\right) \rightarrow S^{p}\left(S_{G}^{p}\right)} & =\left\|M_{A}\right\|_{c b, S_{G}^{p} \rightarrow S_{G}^{p}} \\
& =\left\|M_{t}\right\|_{c b, L^{p}(\operatorname{VN}(G)) \rightarrow L^{p}(\operatorname{VN}(G))} \\
& \leqslant 1 .
\end{aligned}
$$

By Corollary 4.3, we deduce finally that, for any complex polynomial $P$ and all $1<p<\infty$, we have

$$
\left\|P\left(M_{t}\right)\right\|_{L^{p}(\mathrm{VN}(G)) \rightarrow L^{p}(\mathrm{VN}(G))} \leqslant\|P\|_{p, S^{p}\left(S_{G}^{p}\right)}=\|P\|_{p, S^{p}} .
$$

In order to prove the next theorem we need the notion of crossproduct. We refer to $\underline{\mathrm{Str}}$ and $\mathrm{Sun}$ for more information on this concept.

Theorem 4.6 Let $G$ be a discrete group. Let $\mathrm{VN}(G) \stackrel{M_{t}}{\longrightarrow} \mathrm{VN}(G)$ be a unital completely positive Fourier multiplier associated with a real valued function $G \stackrel{t}{\rightarrow} \mathbb{R}$. Then there exists a von Neumann algebra $M$ equipped with a faithful finite normal trace, a unital trace preserving $*$-automorphism $M \stackrel{U}{\rightarrow}$ $M$, a unital normal trace preserving one-to-one $*$-homomorphism $\mathrm{VN}(G) \stackrel{J}{\rightarrow} M$ such that,

$$
\left(M_{t}\right)^{k}=\mathbb{E} U^{k} J
$$

for any integer $k \geqslant 0$, where $M \stackrel{\mathbb{E}}{\rightarrow} V N(G)$ is the canonical faithful normal trace preserving conditional expectation associated with $J$.

Proof : Since the map $\mathrm{VN}(G) \stackrel{M_{t}}{\longrightarrow} \mathrm{VN}(G)$ is completely positive, we can define a positive symmetric bilinear form $\langle\cdot, \cdot\rangle_{\ell} 2, t$ on the real span of the $e_{g}$, where $g \in G$, by:

$$
\left\langle e_{g}, e_{h}\right\rangle_{\ell^{2, t}}=t_{g^{-1} h} .
$$

We denote by $\ell^{2, t}$ the completion of the real pre-Hilbert space obtained by quotient by the corresponding kernel. For all $g \in G$, we denote by $g$ the class of $e_{g}$ in $\ell^{2, t}$. Then, for all $g, h \in G$, we have

$$
\langle g, h\rangle_{\ell^{2, t}}=t_{g^{-1} h} .
$$

For all $g \in G$, it easy to see that there exists a unique isometry $\ell^{2, t} \stackrel{\theta_{g}}{\rightarrow} \ell^{2, t}$ such that for all $h \in G$ we have $\theta_{g}(h)=g h$.

For all $g \in G$, we define the unital trace preserving $*$-automorphism $\alpha(g)=\Gamma_{-1}\left(\theta_{g} \otimes I d_{\ell_{\mathbb{Z}}^{2}}\right)$ :

$$
\begin{array}{ccc}
\alpha(g): \quad \Gamma_{-1}\left(\ell^{2, t} \otimes_{2} \ell_{\mathbb{Z}}^{2}\right) & \longrightarrow & \Gamma_{-1}\left(\ell^{2, t} \otimes_{2} \ell_{\mathbb{Z}}^{2}\right) \\
w(h \otimes v) & \longmapsto & w(g h \otimes v)
\end{array}
$$

The homomorphism $G \stackrel{\alpha}{\rightarrow} \operatorname{Aut}\left(\Gamma_{-1}\left(\ell^{2, t} \otimes_{2} \ell_{\mathbb{Z}}^{2}\right)\right)$ allows us to define the von Neumann algebra

$$
M=\Gamma_{-1}\left(\ell^{2, t} \otimes_{2} \ell_{\mathbb{Z}}^{2}\right) \rtimes_{\alpha} G .
$$

We can identify $\Gamma_{-1}\left(\ell^{2, t} \otimes_{2} \ell_{\mathbb{Z}}^{2}\right)$ as a subalgebra of $M$. We let $J$ the canonical normal unital injective *-homomorphism $\mathrm{VN}(G) \stackrel{J}{\rightarrow} M$. We denote by $\tau$ the faithful finite normal trace on $\Gamma_{-1}\left(\ell^{2, t} \otimes_{2} \ell_{\mathbb{Z}}^{2}\right)$. 
Recall that, for all $g \in G$, the map $\alpha(g)$ is trace preserving. Thus, the trace $\tau$ is $\alpha$-invariant. We equip $M$ with the induced canonical trace $\tau_{M}$. For all $x \in \Gamma_{-1}\left(\ell^{2, t} \otimes_{2} \ell_{\mathbb{Z}}^{2}\right)$ and all $g \in G$, we have

$$
\tau_{M}(x J(\lambda(g)))=\delta_{g, e_{G}} \tau(x)
$$

(see $\left[\mathrm{Str}\right.$ pages 359 and 352 ). If $g, h \in G$ and $v \in \ell_{\mathbb{Z}}^{2}$, we can write the relations of commutation of the crossed product as

$$
J(\lambda(g)) \omega(h \otimes v)=\omega(g h \otimes v) J(\lambda(g)) .
$$

We denote by $M \stackrel{\mathbb{E}}{\rightarrow} \mathrm{VN}(G)$ the canonical faithful normal trace preserving conditional expectation. For all $x \in \Gamma_{-1}\left(\ell^{2, t} \otimes_{2} \ell_{\mathbb{Z}}^{2}\right)$ and all $g \in G$ we have

$$
\mathbb{E}(x J(\lambda(g)))=\tau(x) \lambda(g) .
$$

We define the unital trace preserving $*$-automorphism $\mathscr{S}=\Gamma_{-1}\left(I d_{\ell^{2}, t} \otimes S\right)$ :

$$
\begin{aligned}
\mathscr{S}: \Gamma_{-1}\left(\ell^{2, t} \otimes_{2} \ell_{\mathbb{Z}}^{2}\right) & \longrightarrow \Gamma_{-1}\left(\ell^{2, t} \otimes_{2} \ell_{\mathbb{Z}}^{2}\right) \\
\omega\left(h \otimes e_{n}\right) & \longmapsto \omega\left(h \otimes e_{n+1}\right) .
\end{aligned}
$$

Since $M_{t}$ is unital, $\omega\left(e_{G} \otimes e_{0}\right)$ is a symmetry, i.e. a selfadjoint unitary element. Moreover, for all $g \in G$, we have $\alpha(g) \mathscr{S}=\mathscr{S} \alpha(g)$. Then, by [Sun, Proposition 4.4.4], we can define a unital $*$-automorphism

$$
\begin{aligned}
& U: \quad M \quad \longrightarrow \quad M \\
& x J(\lambda(g)) \longmapsto \omega\left(e_{G} \otimes e_{0}\right) \mathscr{S}(x) J(\lambda(g)) \omega\left(e_{G} \otimes e_{0}\right) .
\end{aligned}
$$

Now, we will show that $U$ preserves the trace. For all $g \in G$ and all $x \in \Gamma_{-1}\left(\ell^{2, t} \otimes_{2} \ell_{\mathbb{Z}}^{2}\right)$, we have

$$
\begin{aligned}
\tau_{M}(U(x J(\lambda(g)))) & =\tau_{M}(\mathscr{S}(x) J(\lambda(g))) \\
& =\delta_{g, e_{G}} \tau(\mathscr{S}(x)) \quad \text { by (4.8) } \\
& =\delta_{g, e_{G}} \tau(x) \\
& =\tau_{M}(x J(\lambda(g))) .
\end{aligned}
$$

We conclude by linearity and normality. It is not hard to see that $J$ preserves the traces.

Now, we will prove that, for any integer $k \geqslant 1$ and any $g \in G$, we have

$$
\begin{aligned}
(4.10) U^{k} & \circ J(\lambda(g)) \\
& =\omega\left(e_{G} \otimes e_{0}\right) \omega\left(e_{G} \otimes e_{1}\right) \cdots \omega\left(e_{G} \otimes e_{k-1}\right) \omega\left(g \otimes e_{k-1}\right) \omega\left(g \otimes e_{k-2}\right) \cdots \omega\left(g \otimes e_{0}\right) J(\lambda(g))
\end{aligned}
$$

by induction on $k$. The statement holds clearly for $k=1$ : if $g \in G$, we have

$$
\begin{aligned}
U \circ J(\lambda(g)) & =\omega\left(e_{G} \otimes e_{0}\right) J(\lambda(g)) \omega\left(e_{G} \otimes e_{0}\right) \\
& =\omega\left(e_{G} \otimes e_{0}\right) \omega\left(g \otimes e_{0}\right) J(\lambda(g)) \quad \text { by (4.9). }
\end{aligned}
$$

Now, assume (4.10). For all $g \in G$, we have

$$
\begin{aligned}
& U^{k+1} \circ J(\lambda(g)) \\
&= U\left(\omega\left(e_{G} \otimes e_{0}\right) \omega\left(e_{G} \otimes e_{1}\right) \cdots \omega\left(e_{G} \otimes e_{k-1}\right) \omega\left(g \otimes e_{k-1}\right) \omega\left(g \otimes e_{k-2}\right) \cdots \omega\left(g \otimes e_{0}\right) J(\lambda(g))\right) \\
&= \omega\left(e_{G} \otimes e_{0}\right) \omega\left(e_{G} \otimes e_{1}\right) \omega\left(e_{G} \otimes e_{2}\right) \cdots \omega\left(e_{G} \otimes e_{k}\right) \omega\left(g \otimes e_{k}\right) \omega\left(g \otimes e_{k-1}\right) \cdots \\
& \omega\left(g \otimes e_{1}\right) J(\lambda(g)) \omega\left(e_{G} \otimes e_{0}\right) \\
&= \omega\left(e_{G} \otimes e_{0}\right) \omega\left(e_{G} \otimes e_{1}\right) \omega\left(e_{G} \otimes e_{2}\right) \cdots \omega\left(e_{G} \otimes e_{k}\right) \omega\left(g \otimes e_{k}\right) \omega\left(g \otimes e_{k-1}\right) \cdots \\
& \omega\left(g \otimes e_{1}\right) \omega\left(g \otimes e_{0}\right) J(\lambda(g)) .
\end{aligned}
$$


We obtained the statement (4.10) for $k+1$. Now let $k \geqslant 1$ and $g \in G$. We define the elements $f_{1}, \ldots, f_{2 k}$ of the Hilbert space $\ell^{2, t} \otimes_{2} \ell_{\mathbb{Z}}^{2}$ by

$$
f_{i}= \begin{cases}e_{G} \otimes e_{i-1} & \text { if } 1 \leqslant i \leqslant k \\ g \otimes e_{2 k-i} & \text { if } k+1 \leqslant i \leqslant 2 k\end{cases}
$$

If $1 \leqslant i \leqslant 2 k$, we have

$$
\begin{aligned}
\left\langle f_{i}, f_{2 k-i+1}\right\rangle_{\ell^{2, t} \otimes_{2} \ell_{\mathbb{Z}}^{2}} & =\left\langle e_{G} \otimes e_{i-1}, g \otimes e_{i-1}\right\rangle_{\ell^{2, t} \otimes_{2} \ell_{\mathbb{Z}}^{2}} \\
& =\left\langle e_{G}, g\right\rangle_{\ell^{2, t}}\left\langle e_{i-1}, e_{i-1}\right\rangle_{\ell_{\mathbb{Z}}^{2}} \\
& =t_{g}
\end{aligned}
$$

By a similar computation, if $1 \leqslant i<j \leqslant 2 k$ with $j \neq 2 k-i+1$, we obtain $\left\langle f_{i}, f_{j}\right\rangle_{\ell^{2, t} \otimes_{2} \ell_{Z}^{2}}=0$. Then, for all $g \in G$, we have

$$
\begin{aligned}
\mathbb{E} U^{k} J(\lambda(g)) & =\mathbb{E}\left(\omega\left(e_{G} \otimes e_{0}\right) \cdots \omega\left(e_{G} \otimes e_{k-1}\right) \omega\left(g \otimes e_{k-1}\right) \cdots \omega\left(g \otimes e_{0}\right) J(\lambda(g))\right) \\
& =\tau\left(\omega\left(e_{G} \otimes e_{0}\right) \cdots \omega\left(e_{G} \otimes e_{k-1}\right) \omega\left(g \otimes e_{k-1}\right) \cdots \omega\left(g \otimes e_{0}\right)\right) \lambda(g) \\
& =\tau\left(\omega\left(f_{1}\right) \omega\left(f_{2}\right) \cdots \omega\left(f_{2 k}\right)\right) \lambda(g) \\
& =\left(\sum_{\mathcal{V} \in \mathcal{P}_{2}(2 k)}(-1)^{c(\mathcal{V})} \prod_{(i, j) \in \mathcal{V}}\left\langle f_{i}, f_{j}\right\rangle_{\ell^{2, t} \otimes_{2} \ell_{Z}^{2}}\right) \lambda(g) \quad \text { by (4.2) } \\
& =\left\langle f_{1}, f_{2 k}\right\rangle_{\ell^{2, t} \otimes_{2} \ell_{\mathbb{Z}}^{2}} \cdots\left\langle f_{k}, f_{k+1}\right\rangle_{\ell^{2, t} \otimes_{2} \ell_{\mathbb{Z}} \lambda(g)} \\
& =\left(t_{g}\right)^{k} \lambda(g) \\
& =T^{k}(\lambda(g)) .
\end{aligned}
$$

(The only non-zero term in the sum of (4.11) is the term with $\mathcal{V}=\{(1,2 k),(2,2 k-1), \ldots,(k, k+1)\}$, which satisfies $c(\mathcal{V})=0$ ). Thus, for any positive integer $k$ (the case $k=0$ is trivial), we conclude that

$$
T^{k}=\mathbb{E} U^{k} J
$$

Corollary 4.7 Let $G$ be a discrete group. Let $\mathrm{VN}(G) \stackrel{M_{t}}{\longrightarrow} \mathrm{VN}(G)$ be a unital completely positive Fourier multiplier which is associated with a real function $G \stackrel{t}{\rightarrow} \mathbb{R}$. Suppose that the von Neumann algebra (4.7) has QWEP. Let $1 \leqslant p \leqslant \infty$. Then, the induced Fourier multiplier $L^{p}(\operatorname{VN}(G)) \stackrel{M_{t}}{\longrightarrow}$ $L^{p}(\mathrm{VN}(G))$ satisfies the noncommutative Matsaev's property.

Proof : This corollary follows from Theorem 4.6, remarks following Lemma 4.1 and Corollary 2.6.

At the light of above corollary, it is important to know when the von Neumann algebra (4.7) has QWEP. If the group $G$ is amenable, this algebra has QWEP by Oza, Proposition 4.1] (or Con, Proposition 6.8]). Now we give an example of non-amenable group $G$ such that this von Neumann algebra has QWEP. We denote by $\mathbb{F}_{n}$ a free group with $n$ generators denoted by $g_{1}, \ldots, g_{n}$ where $1 \leqslant n \leqslant \infty$. We denote by $\mathcal{R}$ the hyperfinite factor of type $\mathrm{II}_{1}$ and by $\mathcal{R}^{\mathcal{U}}$ an ultrapower of $\mathcal{R}$ with respect to a non-trivial ultrafilter $\mathcal{U}$. In order to prove the next theorem we need the notion of amalgamated free product of von Neumann algebras. We refer to [BID] and [Ued] for more information on this concept. Note that, with the notations of the proof of Theorem 4.6, the von Neumann algebra $\Gamma_{-1}\left(\ell^{2, t} \otimes_{2} \ell_{\mathbb{Z}}^{2}\right)$ is $*$-isomorphic to the hyperfinite factor of type $\mathrm{II}_{1}$. 
Proposition 4.8 Suppose $1 \leqslant n \leqslant \infty$. Let $\mathbb{F}_{n} \stackrel{\alpha}{\rightarrow} \operatorname{Aut}(\mathcal{R})$ be a homomorphism. Then the crossed product $\mathcal{R} \rtimes_{\alpha} \mathbb{F}_{n}$ has $Q W E P$.

Proof : First we will show the result for $n=2$. We denote by $\left\langle g_{1}\right\rangle$ and $\left\langle g_{2}\right\rangle$ the subgroups of $\mathbb{F}_{2}$ generated by $g_{1}$ and $g_{2}$ and by $\alpha_{1}$ and $\alpha_{2}$ the restrictions of $\alpha$ to these subgroups. First, we prove that the subalgebras $\mathcal{R} \rtimes_{\alpha_{1}}\left\langle g_{1}\right\rangle$ and $\mathcal{R} \rtimes_{\alpha_{2}}\left\langle g_{2}\right\rangle$ are free with respect to the canonical faithful normal trace preserving conditional expectation $\mathcal{R} \rtimes_{\alpha} \mathbb{F}_{2} \stackrel{\mathbb{E}}{\rightarrow} \mathcal{R}$. We identify $\mathcal{R}$ as a subalgebra of $\mathcal{R} \rtimes_{\alpha} \mathbb{F}_{2}$. We may regard the elements of $\mathcal{R} \rtimes_{\alpha} \mathbb{F}_{2}$ as matrices $\left[\alpha_{r^{-1}}\left(\varpi\left(r t^{-1}\right)\right)\right]_{r, t \in \mathbb{F}_{2}}$ with entries in $\mathcal{R}$ where $\mathbb{F}_{2} \stackrel{\varpi}{\longrightarrow} \mathcal{R}$ is a map. Recall that the conditional expectation $\mathbb{E}$ on $\mathcal{R}$ is given by

$$
\mathbb{E}\left(\left[\alpha_{r^{-1}}\left(\varpi\left(r t^{-1}\right)\right)\right]_{r, t \in \mathbb{F}_{2}}\right)=\varpi\left(e_{\mathbb{F}_{2}}\right) .
$$

Suppose that $i_{1}, \ldots, i_{k} \in\{1,2\}$ are integers such that $i_{1} \neq i_{2}, \ldots, i_{k-1} \neq i_{k}$. For any $1 \leqslant j \leqslant k$, let

$$
A_{j}=\left[\alpha_{r^{-1}}\left(\varpi_{j}\left(r t^{-1}\right)\right)\right]_{r, t \in \mathbb{F}_{2}}
$$

be an element of $\mathcal{R} \rtimes_{\alpha_{i_{j}}}\left\langle g_{i_{j}}\right\rangle$ such that $\mathbb{E}\left(A_{j}\right)=0$ where each $\mathbb{F}_{2} \stackrel{\varpi_{j}}{\longrightarrow} \mathcal{R}$ is a map satisfying $\varpi_{j}(g)=0$ if $g \notin\left\langle g_{i_{j}}\right\rangle$. Then, for all $1 \leqslant j \leqslant k$, we have $\varpi_{j}\left(e_{\mathbb{F}_{2}}\right)=0$. Now, we have

$$
\begin{aligned}
\mathbb{E}\left(A_{1} \cdots A_{k}\right) & =\mathbb{E}\left(\left[\alpha_{r^{-1}}\left(\varpi_{1}\left(r t^{-1}\right)\right)\right]_{r, t \in \mathbb{F}_{2}} \cdots\left[\alpha_{r-1}\left(\varpi_{k}\left(r t^{-1}\right)\right)\right]_{r, t \in \mathbb{F}_{2}}\right) \\
& =\sum_{L^{1}, \ldots, l_{k-1} \in \mathbb{F}_{2}} \varpi_{1}\left(l_{1}^{-1}\right) \alpha_{l_{1}^{-1}}\left(\varpi_{2}\left(l_{1} l_{2}^{-1}\right)\right) \cdots \alpha_{l_{k-2}^{-1}}\left(\varpi_{2}\left(l_{k-2} l_{k-1}^{-1}\right)\right) \alpha_{l_{k-1}^{-1}}\left(\varpi_{k}\left(l_{k-1}\right)\right) \\
& =0 .
\end{aligned}
$$

Thus the von Neumann algebra $\mathcal{R} \rtimes_{\alpha} \mathbb{F}_{2}$ decomposes as an amalgamated free product of $\mathcal{R} \rtimes_{\alpha_{1}}\left\langle g_{1}\right\rangle$ and $\mathcal{R} \rtimes_{\alpha_{2}}\left\langle g_{2}\right\rangle$ over $\mathcal{R}$. Moreover, the groups $\left\langle g_{1}\right\rangle$ and $\left\langle g_{2}\right\rangle$ are commutative, hence amenable. We have already point out that the crossed product of the hyperfinite factor $\mathcal{R}$ by an amenable group has QWEP. Then the von Neumann algebras $\mathcal{R} \rtimes_{\alpha_{1}}\left\langle g_{1}\right\rangle$ and $\mathcal{R} \rtimes_{\alpha_{2}}\left\langle g_{2}\right\rangle$ are QWEP. Moreover, by [Bla, page 283], these von Neumann algebras have a separable predual. By [Kir, Theorem 1.4], we deduce that these von Neumann algebras are embeddable into $\mathcal{R}^{\mathcal{U}}$. Now, the theorem stating in [BDJ, Corollary 4.5] says that, for finite von Neumann algebras with separable preduals, being embeddable into $\mathcal{R}^{\mathcal{U}}$ is stable under amalgamated free products over a hyperfinite von Neumann algebra. Thus we deduce that $\mathcal{R} \rtimes_{\alpha} \mathbb{F}_{2}$ is embeddable into $\mathcal{R}^{\mathcal{U}}$, which is equivalent to QWEP, by [Kir, Theorem 1.4], since $\mathcal{R} \rtimes_{\alpha} \mathbb{F}_{2}$ has a separable predual. Induction then gives the case when $2 \leqslant n<\infty$, and the case $n=\infty$ then follows since, by [Oza, Proposition 4.1], QWEP is preserved by taking the weak* closure of increasing unions of von Neumann algebras.

We pass to maps arising in the second quantization in the context of BKS].

Proposition 4.9 Suppose $1<p<\infty$ and $-1 \leqslant q<1$. Let $H$ be a real Hilbert space and $H \stackrel{T}{\rightarrow}$ $H$ a contraction. Then the induced map $L^{p}\left(\Gamma_{q}(H)\right) \stackrel{M_{t}}{\longrightarrow} L^{p}\left(\Gamma_{q}(H)\right)$ satisfies the noncommutative Matsaev's property.

Proof : There exists an orthogonal dilation $K \stackrel{U}{\rightarrow} K$ de $H \stackrel{T}{\rightarrow} H$. We denote by $H \stackrel{J}{\rightarrow} K$ the embedding of $H$ in $K$ and $K \stackrel{Q}{\longrightarrow} H$ the projection of $K$ on $H$. The map $\Gamma_{q}(K) \stackrel{\Gamma(J)}{\longrightarrow} \Gamma_{q}(K)$ is a unital injective normal trace preserving $*$-homomorphism. The map $\Gamma_{q}(H) \stackrel{\Gamma(U)}{\longrightarrow} \Gamma_{q}(K)$ is a unital 
trace preserving *-automorphism. The map $\Gamma_{q}(K) \stackrel{\Gamma(Q)}{\longrightarrow} \Gamma_{q}(H)$ is the canonical faithful normal unital trace preserving conditional expectation of $\Gamma_{q}(K)$ on $\Gamma_{q}(H)$. Moreover, we have for any integer $k$

$$
\Gamma_{q}(T)^{k}=\Gamma_{q}(P) \Gamma_{q}(U)^{k} \Gamma_{q}(J) .
$$

We conclude with Theorem 4.6, remarks following Lemma 4.1. Corollary 2.6 and by using the fact that, by $\mathrm{Nou}]$, the von Neumann algebra $\Gamma_{q}(H)$ has QWEP.

In order to state more easily our following result we need to define the following property. Let $M$ be a von Neumann algebra. Suppose that $M \stackrel{T}{\rightarrow} M$ is a linear map.

Property 4.10 There exists a von Neumann algebra $N$ with QWEP equipped with a normal faithful finite trace on $N$, a unital trace preserving *-automorphism $N \stackrel{U}{\rightarrow} N$, a unital injective normal trace preserving $*$-homomorphism $M \stackrel{J}{\rightarrow} N$ such that,

$$
T^{k}=\mathbb{E} U^{k} J
$$

for any integer $k \geqslant 0$, where $M \stackrel{\mathbb{E}}{\rightarrow} V N(G)$ is the canonical faithful normal trace preserving conditional expectation associated with $J$.

This property is stable under free product. Indeed, one can prove the next proposition with an argument similar to that used in the proof of [JMX, Lemma 10.4] and by using [BDJ, Corollary 4.5] and [Kir, Theorem 1.4].

Proposition 4.11 Let $M_{1}$ and $M_{2}$ be von Neumann algebras with separable preduals equipped with normal faithful finite traces $\tau_{1}$ and $\tau_{2}$. Let $M_{1} \stackrel{T_{1}}{\rightarrow} M_{1}$ and $M_{2} \stackrel{T_{2}}{\longrightarrow} M_{2}$ be linear maps. If $T_{1}$ and $T_{2}$ satisfy Property 4.10, their free product

$$
\left(M_{1}, \tau_{1}\right) \approx\left(M_{2}, \tau_{2}\right) \stackrel{T_{1} \mp T_{2}}{\longrightarrow}\left(M_{1}, \tau_{1}\right) \bar{*}\left(M_{2}, \tau_{2}\right)
$$

also satisfies Property 4.10

Thus the above proposition allows us to construct other examples of contractions satisfying the noncommutative Matsaev's property.

\section{The case of semigroups}

Suppose $1 \leqslant p<\infty$. We denote by $\left(\mathcal{T}_{t}\right)_{t \geqslant 0}$ the translation semigroup on $L^{p}(\mathbb{R})$, where $\mathcal{T}_{t}(f)(s)=$ $f(s-t)$ if $f \in L^{p}(\mathbb{R})$ and $s, t \in \mathbb{R}$. This semigroup $\left(\mathcal{T}_{t}\right)_{t \geqslant 0}$ is a $C_{0}$-semigroup of contractions.

Let $\left(T_{t}\right)_{t \geqslant 0}$ be a $C_{0}$-semigroup of contractions on a Banach space $X$. For all $b \in L^{1}(\mathbb{R})$ with support in $\mathbb{R}^{+}$, it is easy to see that the linear operator

$$
\begin{array}{rllc}
\int_{0}^{+\infty} b(t) T_{t} d t: \quad X & \longrightarrow & X \\
x & \longmapsto \int_{0}^{+\infty} b(t) T_{t} x d t
\end{array}
$$

is well-defined and bounded. Moreover, we have

$$
\left\|\int_{0}^{+\infty} b(t) T_{t} d t\right\|_{X \rightarrow X} \leqslant\|b\|_{L^{1}(\mathbb{R})} .
$$

Now, let us state a question for semigroups which is analogue to Matsaev's Conjecture 1.1 . 
Question 5.1 Suppose $1<p<\infty, p \neq 2$. Let $\left(T_{t}\right)_{t \geqslant 0}$ be a $C_{0}$-semigroup of contractions on a $L^{p}$-space $L^{p}(\Omega)$ of a measure space $\Omega$. Do we have the following estimate

$$
\left\|\int_{0}^{+\infty} b(t) T_{t} d t\right\|_{L^{p}(\Omega) \rightarrow L^{p}(\Omega)} \leqslant\left\|\int_{0}^{+\infty} b(t) \mathcal{T}_{t} d t\right\|_{L^{p}(\mathbb{R}) \rightarrow L^{p}(\mathbb{R})}
$$

for all $b \in L^{1}(\mathbb{R})$ with support in $\mathbb{R}^{+}$?

We pass to the noncommutative case. We can state the following noncommutative analogue of Question 5.1 .

Question 5.2 Suppose $1<p<\infty, p \neq 2$. Let $\left(T_{t}\right)_{t \geqslant 0}$ be a $C_{0}$-semigroup of contractions on a noncommutative $L^{p}$-space $L^{p}(M)$. Do we have the following estimate

$$
\left\|\int_{0}^{+\infty} b(t) T_{t} d t\right\|_{L^{p}(M) \rightarrow L^{p}(M)} \leqslant\left\|\int_{0}^{+\infty} b(t) \mathcal{T}_{t} d t\right\|_{c b, L^{p}(\mathbb{R}) \rightarrow L^{p}(\mathbb{R})}
$$

for all $b \in L^{1}(\mathbb{R})$ with support in $\mathbb{R}^{+}$?

For all $b \in L^{1}(\mathbb{R})$ with support in $\mathbb{R}^{+}$, it is clear that $C_{b}=\int_{0}^{+\infty} b(t) \mathcal{T}_{t} d t$. Moreover, for all $b \in L^{1}(\mathbb{R})$, we have

$$
\left\|C_{b}\right\|_{L^{1}(\mathbb{R}) \rightarrow L^{1}(\mathbb{R})}=\left\|C_{b}\right\|_{c b, L^{1}(\mathbb{R}) \rightarrow L^{1}(\mathbb{R})}=\|b\|_{L^{1}(\mathbb{R})} .
$$

Consequently, the inequalities (5.1) and (5.2) hold true for $p=1$.

In [CoW, page 25], it is proved that the $C_{0}$-semigroups of positive contractions satisfy inequality (5.1). Using [Pel1, Theorem 3] and the same method, we can generalize this result to $C_{0}$-semigroups of operators which admit a contractive majorant. Now, we adapt this method in order to give a link between Question 5.2 and Question 1.3

Theorem 5.3 Suppose $1<p<\infty$. Let $\left(T_{t}\right)_{t \geqslant 0}$ be a $C_{0}$-semigroup of contractions on a noncommutative $L^{p}$-space $L^{p}(M)$ such that each $L^{p}(M) \stackrel{T_{t}}{\longrightarrow} L^{p}(M)$ satisfies the noncommutative Matsaev's property. Then the semigroup $\left(T_{t}\right)_{t \geqslant 0}$ satisfies inequality (5.2).

Proof : It is not hard to see that it suffices to prove this in the case when $b$ has compact support. Now we define the sequence $\left(a_{n}\right)_{n \geqslant 1}$ of complex sequences indexed by $\mathbb{Z}$ as in the proof of Theorem 3.5. Let $n \geqslant 1$. Observe that if $\mathbb{R}^{+} \stackrel{f}{\rightarrow} L^{p}(M)$ is continuous and piecewise affine with nodes at $\frac{k}{n}$ then

$$
\int_{0}^{+\infty} b(t) f(t) d t=\sum_{k=0}^{+\infty} a_{n, k} f\left(\frac{k}{n}\right) .
$$

Let $x \in L^{p}(M)$. Let $\mathbb{R}^{+} \stackrel{f_{n}}{\longrightarrow} L^{p}(M)$ be the continuous and piecewise affine function with nodes at $\frac{k}{n}$ such that $f_{n}\left(\frac{k}{n}\right)=\left(T_{\frac{1}{n}}\right)^{k} x$. Since the map $t \mapsto T_{t} x$ is uniformly continuous on compacts of $\mathbb{R}^{+}$we have

$$
\begin{aligned}
\left\|\int_{0}^{+\infty} b(t) T_{t} x d t-\sum_{k=0}^{+\infty} a_{n, k}\left(T_{\frac{1}{n}}\right)^{k} x\right\|_{L^{p}(M)} & =\left\|\int_{0}^{+\infty} b(t) T_{t} x d t-\sum_{k=0}^{+\infty} a_{n, k} f_{n}\left(\frac{k}{n}\right)\right\|_{L^{p}(M)} \\
& =\left\|\int_{0}^{+\infty} b(t)\left(T_{t} x-f_{n}(t)\right) d t\right\|_{L^{p}(M)} \underset{n \rightarrow+\infty}{\stackrel{\longrightarrow}{\longrightarrow}} .
\end{aligned}
$$


We deduce that

$$
\sum_{k=0}^{+\infty} a_{n, k}\left(T_{\frac{1}{n}}\right)^{k} \underset{n \rightarrow+\infty}{\stackrel{\text { so }}{\longrightarrow}} \int_{0}^{+\infty} b(t) T_{t} d t .
$$

By the commutative diagram of the proof of Theorem 3.5. we have for any integer $n \geqslant 1$

$$
\left\|C_{a_{n}}\right\|_{c b, \ell_{\mathbb{Z}}^{p} \rightarrow \ell_{\mathbb{Z}}^{p}} \leqslant\left\|C_{b}\right\|_{c b, L^{p}(\mathbb{R}) \rightarrow L^{p}(\mathbb{R})} .
$$

Finally, by the strongly lower semicontinuity of the norm, we obtain that

$$
\begin{aligned}
\left\|\int_{0}^{+\infty} b(t) T_{t} d t\right\|_{L^{p}(M) \rightarrow L^{p}(M)} & \leqslant \liminf _{n \rightarrow+\infty}\left\|\sum_{k=1}^{+\infty} a_{n, k}\left(T_{\frac{1}{n}}\right)^{k}\right\|_{L^{p}(M) \rightarrow L^{p}(M)} \\
& \leqslant \liminf _{n \rightarrow+\infty}\left\|C_{a_{n}}\right\|_{c b, \ell_{\mathbb{Z}}^{p} \rightarrow \ell_{\mathbb{Z}}^{p}} \\
& =\left\|C_{b}\right\|_{c b, L^{p}(\mathbb{R}) \rightarrow L^{p}(\mathbb{R}) .}
\end{aligned}
$$

The first consequence of this theorem is that inequality (5.2) holds true for $p=2$. Now, we list some natural examples of semigroups which satisfy inequality (5.2) by our results, using this theorem.

Semigroups of Schur multipliers. Let $\left(T_{t}\right)_{t \geqslant 0}$ a $\mathrm{w}^{*}$-semigroup of selfadjoint contractive Schur multipliers on $B\left(\ell_{I}^{2}\right)$. If $1 \leqslant p<\infty$ and $t \geqslant 0$, the map $B\left(\ell_{I}^{2}\right) \stackrel{T_{t}}{\longrightarrow} B\left(\ell_{I}^{2}\right)$ induces a contraction $S_{I}^{p} \stackrel{T_{t}}{\longrightarrow} S_{I}^{p}$. Using [JMX, Remark 5.2], it is easy to see that we obtain a $C_{0}$-semigroup of contractions $S_{I}^{p} \stackrel{T_{t}}{\longrightarrow} S_{I}^{p}$ which satisfies inequality (5.2).

Semigroups of Fourier multipliers on an amenable group. Let $G$ be an amenable group. Let $\left(T_{t}\right)_{t \geqslant 0} \mathrm{a} \mathrm{w}^{*}$-semigroup of selfadjoint contractive Fourier multipliers on $V N(G)$. If $1 \leqslant p<\infty$ and $t \geqslant 0$, the map $V N(G) \stackrel{T_{t}}{\longrightarrow} V N(G)$ induces a contraction $L^{p}(V N(G)) \stackrel{T_{t}}{\longrightarrow} L^{p}(V N(G))$. We obtain a $C_{0}$-semigroup of contractions $L^{p}(V N(G)) \stackrel{T_{t}}{\longrightarrow} L^{p}(V N(G))$ which satisfies inequality (5.2).

Noncommutative Poisson semigroup. Let $n \geqslant 1$ be an integer. Recall that $\mathbb{F}_{n}$ denotes a free group with $n$ generators denoted by $g_{1}, \ldots, g_{n}$. A semigroup on $L^{p}\left(V N\left(\mathbb{F}_{n}\right)\right)$ induced by a $w^{*}$ semigroup of selfadjoint completely positive unital Fourier multipliers on $V N\left(\mathbb{F}_{n}\right)$ satisfies inequality (5.2). An example is provided by the following semigroup. Any $g \in \mathbb{F}_{n}$ has a unique decomposition of the form

$$
g=g_{i_{1}}^{k_{1}} g_{i_{2}}^{k_{2}} \cdots g_{i_{l}}^{k_{l}}
$$

where $l \geqslant 0$ is an integer, each $i_{j}$ belongs to $\{1, \ldots, n\}$, each $k_{j}$ is a non-zero integer, and $i_{j} \neq i_{j+1}$ if $1 \leqslant j \leqslant l-1$. The case when $l=0$ corresponds to the unit element $g=e_{\mathbb{F}_{n}}$. By definition, the length of $g$ is defined as

$$
|g|=\left|k_{1}\right|+\cdots+\left|k_{l}\right|
$$

This is the number of factors in the above decomposition of $g$. For any nonnegative real number $t \geqslant 0$, we have a normal unital completely positive selfadjoint map

$$
\begin{aligned}
T_{t}: \mathrm{VN}\left(\mathbb{F}_{n}\right) & \longrightarrow \\
\lambda(g) & \longmapsto e^{-t|g|} \lambda(g) .
\end{aligned}
$$

These maps define a $\mathrm{w}^{*}$-semigroup $\left(T_{t}\right)_{t \geqslant 0}$ called the noncommutative Poisson semigroup (see [JMX] for more information). If $1 \leqslant p<\infty$, this semigroup defines a $C_{0}$-semigroup of contractions $L^{p}\left(\mathrm{VN}\left(\mathbb{F}_{n}\right)\right) \stackrel{T_{t}}{\longrightarrow} L^{p}\left(\mathrm{VN}\left(\mathbb{F}_{n}\right)\right)$ which satisfies inequality (5.2). 
$q$-Ornstein-Uhlenbeck semigroup. Suppose $-1 \leqslant q<1$. Let $H$ be a real Hilbert space and let $\left(a_{t}\right)_{t \geqslant 0}$ be a $C_{0}$-semigroup of contractions on $H$. For any $t \geqslant 0$, let $T_{t}=\Gamma_{q}\left(a_{t}\right)$. Then $\left(T_{t}\right)_{\geqslant 0}$ is a $\mathrm{w}^{*}$-semigroup of normal unital completely positive maps on the von Neumann algebra $\Gamma_{q}(H)$. If $1 \leqslant p<\infty$, this semigroup defines a $C_{0}$-semigroup of contractions $L^{p}\left(\Gamma_{q}(H)\right) \stackrel{T_{t}}{\longrightarrow} L^{p}\left(\Gamma_{q}(H)\right)$ (see [JMX] for more information). This semigroup satisfies inequality (5.2).

In the case where $a_{t}=e^{-t} I_{H}$, the semigroup $\left(T_{t}\right)_{\geqslant 0}$ is the so-called $q$-Ornstein-Uhlenbeck semigroup.

Modular semigroups. The $C_{0}$-semigroups of isometries satisfy inequality (5.2). Examples are provided by modular automorphisms semigroups. Here we use noncommutative $L^{p}$-spaces of a von Neumann algebra equipped with a distinguished normal faithful state, constructed by Haagerup. We refer to $[\mathrm{PiX}]$, and the references therein, for more information on these spaces. Let $M$ be a von Neumann algebra with QWEP equipped with a normal faithful state $M \stackrel{\varphi}{\rightarrow} \mathbb{C}$. Let $\left(\sigma_{t}^{\varphi}\right)_{t \in \mathbb{R}}$ be the modular group of $\varphi$. If $1 \leqslant p<\infty$, it is well known that $\left(\sigma_{t}^{\varphi}\right)_{t \geqslant 0}$ induces a $C_{0}$-semigroup of isometries $L^{p}(M) \stackrel{\sigma_{t}^{\varphi}}{\longrightarrow} L^{p}(M)$ (see [JuX]). This semigroup satisfies inequality (5.2).

In the light of Theorem 4.2 it is natural to ask for dilations of unital selfadjoint completely positive semigroups of Schur multipliers. Actually, these semigroups admit a description which allows us to construct a such dilation.

Proposition 5.4 Suppose that $A$ is a matrix of $\mathbb{M}_{I}$. For all $t \geqslant 0$, let $T_{t}$ be the unbounded Schur multipliers on $B\left(\ell_{I}^{2}\right)$ associated with the matrix

$$
\left[e^{-t a_{i j}}\right]_{i, j \in I}
$$

Then the semigroup $\left(T_{t}\right)_{t \geqslant 0}$ extends to a semigroup of selfadjoint unital completely positive Schur multipliers $B\left(\ell_{I}^{2}\right) \stackrel{T_{t}}{\longrightarrow} B\left(\ell_{I}^{2}\right)$ if and only if there exists a Hilbert space $H$ and a family $\left(\alpha_{i}\right)_{i \in I}$ of elements of $H$ such that for all $t \geqslant 0$ the Schur multiplier $B\left(\ell_{I}^{2}\right) \stackrel{T_{t}}{\longrightarrow} B\left(\ell_{I}^{2}\right)$ is associated with the matrix

$$
\left[e^{-t\left\|\alpha_{i}-\alpha_{j}\right\|_{H}^{2}}\right]_{i, j \in I} \text {. }
$$

In this case, the Hilbert space may be chosen as a real Hilbert space. Moreover, $\left(T_{t}\right)_{t \geqslant 0}$ is a $w^{*}$-semigroup.

Proof : Now say that each $T_{t}$ is a selfadjoint unital completely positive contraction means that for all $t>0$, the matrix (5.3) defines a real-valued positive definite kernel on $I \times I$ in the sense of [BCR, Chapter 3, Definition 1.1] such that for all $i \in I$ we have $a_{i i}=0$. Now, the theorem of Schoenberg [BCR, Theorem 2.2] affirms that if $\psi$ is a kernel then $e^{-t \psi}$ is a positive definite kernel for all $t>0$ if and only if $\psi$ is a negative definite kernel. Consequently, the last assertion is equivalent to the fact that $A$ defines a real-valued negative definite kernel which vanishes on the diagonal of $I \times I$. Finally, the characterization of real-valued definite negative kernel of [BCR, Proposition 3.2] gives the equivalence with the required description.

The assertion concerning the choice of the Hilbert space is clear. Finally, using [JMX, Remark $5.2]$, it is easy to see that $\left(T_{t}\right)_{t \geqslant 0}$ is a $\mathrm{w}^{*}$-semigroup.

The next proposition is inspired by the work [JuX].

Proposition 5.5 Let $\left(T_{t}\right)_{t \geqslant 0}$ be a $w^{*}$-semigroup of selfadjoint unital completely positive Schur multipliers on $B\left(\ell_{I}^{2}\right)$. Then, there exists a hyperfinite von Neumann algebra $M$ equipped with a semifinite 
normal faithful trace, a $w^{*}$-semigroup $\left(U_{t}\right)_{t \geqslant 0}$ of unital trace preserving ${ }^{*}$-automorphisms of $M$, a unital trace preserving one-to-one normal $*$-homomorphism $B\left(\ell_{I}^{2}\right) \stackrel{J}{\rightarrow} M$ such that

$$
T_{t}=\mathbb{E} U_{t} J
$$

for any $t \geqslant 0$, where $M \stackrel{\mathbb{E}}{\rightarrow} B\left(\ell_{I}^{2}\right)$ is the canonical faithful normal trace preserving conditional expectation associated with $J$.

Proof : By Proposition 5.4, there exists a real Hilbert space $H$ and a family $\left(\alpha_{j}\right)_{j \in I}$ of elements of $H$ such that, for all $t \geqslant 0$, the Schur multiplier $B\left(\ell_{I}^{2}\right) \stackrel{T_{t}}{\rightarrow} B\left(\ell_{I}^{2}\right)$ is associated with the matrix

$$
\left[e^{-t\left\|\alpha_{j}-\alpha_{k}\right\|_{H}^{2}}\right]_{j, k \in I} \text {. }
$$

Let $\mu$ be a gaussian measure on $H$, i.e. a probability space $(\Omega, \mu)$ together with a measurable function $\Omega \stackrel{w}{\rightarrow} H$ such that, for all $h \in H$, we have

$$
e^{-\|h\|_{H}^{2}}=\int_{\Omega} e^{i\langle h, w(\omega)\rangle_{H}} d \mu(\omega)
$$

where $i^{2}=-1$. We define the von Neumann algebra $M=L^{\infty}(\Omega) \otimes B\left(\ell_{I}^{2}\right)$. Note that $M$ is a hyperfinite von Neumann algebra. We equip the von Neumann algebra $M$ with the faithful semifinite normal trace $\tau_{M}=\int_{\Omega} \cdot d \mu \otimes \operatorname{Tr}$. Note that, by [Sak, Theorem 1.22.13], we have a $*$-isomorphism $M=L^{\infty}\left(\Omega, B\left(\ell_{I}^{2}\right)\right)$. We define the canonical injective normal unital $*$-homomorphism

$$
\begin{array}{ccc}
J: B\left(\ell_{I}^{2}\right) & \longrightarrow & L^{\infty}(\Omega) \bar{\otimes} B\left(\ell_{I}^{2}\right) \\
x & \longmapsto & 1 \otimes x
\end{array}
$$

It is clear that the map $J$ preserves the traces. We denote by $M \stackrel{\mathbb{E}}{\rightarrow} B\left(\ell_{I}^{2}\right)$ the canonical faithful normal trace preserving conditional expectation of $M$ onto $B\left(\ell_{I}^{2}\right)$. For all $\omega \in \Omega$ and $t>0$ let $D_{t}(\omega)$ be the diagonal matrix of $B\left(\ell_{I}^{2}\right)$ defined by

$$
D_{t}(\omega)=\left[\delta_{j, k} e^{i \sqrt{t}\left\langle\alpha_{j}, w(\omega)\right\rangle_{\ell_{I}^{2}}}\right]_{j, k \in I} .
$$

Note that, for all $t>0$, the map $\Omega \stackrel{D_{t}}{\longrightarrow} B(H)$ defines an unitary element of $L^{\infty}\left(\Omega, B\left(\ell_{I}^{2}\right)\right)$. Now, for all $t \geqslant 0$ we define the linear map

$$
\begin{array}{ccc}
U_{t}: L^{\infty}\left(\Omega, B\left(\ell_{I}^{2}\right)\right) & \longrightarrow L^{\infty}\left(\Omega, B\left(\ell_{I}^{2}\right)\right) \\
f & D_{t} f D_{t}^{*} .
\end{array}
$$

If $t \geqslant 0$, it is easy to see that the map $U_{t}$ is a trace preserving $*$-automorphism of $M$. For all $x \in B\left(\ell_{I}^{2}\right)$, we have

$$
\begin{aligned}
\mathbb{E} U_{t} J(x) & =\mathbb{E} U_{t}(1 \otimes x) \\
& =\int_{\Omega} D_{t}(\omega)(1 \otimes x) D_{t}(\omega)^{*} d \mu(\omega) \\
& =\int_{\Omega}\left[e^{i \sqrt{t}\left\langle\alpha_{j}-\alpha_{k}, w(\omega)\right\rangle_{H}} x_{j k}\right]_{j, k \in I} d \mu(\omega) \\
& =\left[e^{-t\left\|\alpha_{j}-\alpha_{k}\right\|_{H}^{2}} x_{j k}\right]_{j, k \in I} \\
& =T_{t}(x) .
\end{aligned}
$$


Thus, for all $t \geqslant 0$, we have

$$
T_{t}=\mathbb{E} U_{t} J
$$

The assertion concerning the regularity of the semigroup is easy and left to the reader.

In the same vein, it is not difficult to construct a dilation of the noncommutative Poisson semigroup. The result was already known to F. Lust-Piquard. Moreover, it is easy to dilate the $C_{0}$-semigroups of contractions $L^{p}\left(\Gamma_{q}(H)\right) \stackrel{\Gamma_{q}\left(a_{t}\right)}{\longrightarrow} L^{p}\left(\Gamma_{q}(H)\right)$, with [SNF, Theorem 8.1].

Finally, we have the next result analogue to Corollary 2.6. One can prove this proposition with a similar argument.

Proposition 5.6 Suppose $1<p<\infty$. Let $\left(T_{t}\right)_{t \geqslant 0}$ be a $C_{0}$-semigroup of contractions on a noncommutative $L^{p}$-space $L^{p}(M)$. Suppose that there exists a noncommutative $L^{p}$-space $L^{p}(N)$ where $N$ has $Q W E P$, a $C_{0}$-semigroup $\left(U_{t}\right)_{t \geqslant 0}$ of isometric operators on $L^{p}(N)$, an isometric embedding $L^{p}(M) \stackrel{J}{\rightarrow} L^{p}(N)$ and a contractive map $L^{p}(N) \stackrel{Q}{\rightarrow} L^{p}(M)$ such that,

$$
T_{t}=Q U_{t} J
$$

for any $t \geqslant 0$. Then, for all $b \in L^{1}(\mathbb{R})$ with support in $\mathbb{R}^{+}$, we have the estimate

$$
\left\|\int_{0}^{+\infty} b(t) T_{t} d t\right\|_{L^{p}(M) \rightarrow L^{p}(M)} \leqslant\left\|\int_{0}^{+\infty} b(t) \mathcal{T}_{t} d t\right\|_{c b, L^{p}(\mathbb{R}) \rightarrow L^{p}(\mathbb{R})} .
$$

Moreover, if $L^{p}(N)$ is a commutative $L^{p}$-space $L^{p}(\Omega)$, we have, for all $b \in L^{1}(\mathbb{R})$ with support in $\mathbb{R}^{+}$, the estimate

$$
\left\|\int_{0}^{+\infty} b(t) T_{t} d t\right\|_{L^{p}(\Omega) \rightarrow L^{p}(\Omega)} \leqslant\left\|\int_{0}^{+\infty} b(t) \mathcal{T}_{t} d t\right\|_{L^{p}(\mathbb{R}) \rightarrow L^{p}(\mathbb{R})} .
$$

This proposition allows us to give alternate proofs for some results of this section. By example, using SNF, Theorem 8.1] of dilation of $C_{0}$-semigroups on a Hilbert space, we deduce that the both inequalities (5.1) and (5.2) are true for $p=2$. By using [Fen], we see that the $C_{0}$-semigroups of operators which admit a contractive majorant satisfy inequality (5.1), for $1<p<\infty$.

\section{$6 \quad$ Final remarks}

We begin by observing that the inequalities (1.1) and (1.4) are true for any complex polynomial $P$ of degree 1 and any contraction $T$. Indeed, suppose that $P(z)=a+b z$, then it is easy to see that $\|P\|_{2}=|a|+|b|$. Thus, for all $1 \leqslant p \leqslant \infty$, we have $\|P\|_{p}=\|P\|_{p, S^{p}}=|a|+|b|$.

Now we will determine the real polynomials of higher degree with a similar property.

Proposition 6.1 Let $P=\sum_{k=0}^{n} a_{k} z^{k}$ be a real polynomial such that $a_{k} \neq 0$ for any $0 \leqslant k \leqslant n$. The following assertions are equivalent.

1. For all $1<p<\infty$, we have $\|P\|_{p}=\sum_{k=0}^{n}\left|a_{k}\right|$. 
2. For all $1<p<\infty$, we have $\|P\|_{p, S^{p}}=\sum_{k=0}^{n}\left|a_{k}\right|$.

3. There exists $1<p<\infty$ such that $\|P\|_{p}=\sum_{k=0}^{n}\left|a_{k}\right|$.

4. There exists $1<p<\infty$ such that $\|P\|_{p, S^{p}}=\sum_{k=0}^{n}\left|a_{k}\right|$.

5. The coefficients $a_{k}$ have the same sign or the signs of the $a_{k}$ are alternating (i.e. for any integer $0 \leqslant k \leqslant n-1$ we have $\left.a_{k} a_{k+1} \leqslant 0\right)$.

In this case, for the polynomial $P$ and any contraction $T$, the inequalities (1.1) and (1.4) are true.

Proof : First we will show that $\|P\|_{2}=\sum_{k=0}^{n}\left|a_{k}\right|$ is equivalent to the last assertion. Recall that $\|P\|_{2}=\sup _{|z|=1}|P(z)|$. On the one hand, for all $0 \leqslant \theta \leqslant 2 \pi$, we have

$$
\begin{aligned}
& \left|\sum_{k=0}^{n} a_{k} e^{k i \theta}\right|^{2}=\left(\sum_{k=0}^{n} a_{k} \cos (k \theta)\right)^{2}+\left(\sum_{k=0}^{n} a_{k} \sin (k \theta)\right)^{2} \\
& =\sum_{k=0}^{n} a_{k}^{2} \cos ^{2}(k \theta)+2 \sum_{0 \leqslant k<l \leqslant n} a_{k} a_{l} \cos (k \theta) \cos (l \theta)+\sum_{k=0}^{n} a_{k}^{2} \sin ^{2}(k \theta) \\
& \quad+2 \sum_{0 \leqslant k<l \leqslant n} a_{k} a_{l} \sin (k \theta) \sin (l \theta) \\
& =\sum_{k=0}^{n} a_{k}^{2}+2 \sum_{0 \leqslant k<l \leqslant n} a_{k} a_{l} \cos ((k-l) \theta) .
\end{aligned}
$$

On the other hand, we have the equality

$$
\left(\sum_{k=0}^{n}\left|a_{k}\right|\right)^{2}=\sum_{k=0}^{n} a_{k}^{2}+2 \sum_{0 \leqslant k<l \leqslant n}\left|a_{k} a_{l}\right| .
$$

Then $P$ satisfies $\|P\|_{2}=\sum_{k=0}^{n}\left|a_{k}\right|$ if and only if

$$
\sum_{0 \leqslant k<l \leqslant n} a_{k} a_{l} \cos ((k-l) \theta)=\sum_{0 \leqslant k<l \leqslant n}\left|a_{k} a_{l}\right| .
$$

This last assertion means that for all $0 \leqslant k<l \leqslant n$ we have $\cos ((k-l) \theta)=\operatorname{sign}\left(a_{k} a_{l}\right)$. It is easy to see that this last assertion is equivalent to the assertion 5 .

Now, it is trivial that the equality $\|P\|_{2}=\sum_{k=0}^{n}\left|a_{k}\right|$ implies the assertions 1 and 2 , that 1 implies 3 and that 2 implies 4 . Now we show that 4 implies $\|P\|_{2}=\sum_{k=0}^{n}\left|a_{k}\right|$. By interpolation, we have

$$
\|P\|_{\infty, S_{\infty}}=\sum_{k=0}^{n}\left|a_{k}\right|=\|P\|_{p, S^{p}} \leqslant\left(\|P\|_{\infty, S_{\infty}}\right)^{1-\frac{2}{p}}\left(\|P\|_{2, S_{2}}\right)^{\frac{2}{p}} .
$$

Moreover, it is easy to see that $\|P\|_{2}=\|P\|_{2, S_{2}}$. Then we obtain

$$
\left(\|P\|_{\infty, S_{\infty}}\right)^{\frac{2}{p}} \leqslant\left(\|P\|_{2, S_{2}}\right)^{\frac{2}{p}}=\left(\|P\|_{2}\right)^{\frac{2}{p}} .
$$


And finally we have

$$
\sum_{k=0}^{n}\left|a_{k}\right|=\|P\|_{\infty, S_{\infty}} \leqslant\|P\|_{2}
$$

The proof that the assertion 3 implies $\|P\|_{2}=\sum_{k=0}^{n}\left|a_{k}\right|$ is similar.

Acknowledgement. I wish to thank my thesis advisor Christian Le Merdy for his support and advice, Eric Ricard for fruitful discussions and Jesse Peterson for suggest me the proof of Proposition 4.8

\section{References}

[AbA] Y. Abramovich and C. Aliprantis. An invitation to operator theory. American Mathematical Society, Providence, 2002.

[AkS] M. Akcoglu and L. Sucheston. Dilations of positive contractions on $L_{p}$ spaces. Canad. Math. Bull. 20, no. 3, 285-292, 1977.

[ALM] C. Arhancet and C. Le Merdy. Dilation of Ritt operators on $L^{p}$-spaces. Preprint, arXiv:1106.1513

[Arh] C. Arhancet. Unconditionality, Fourier multipliers and Schur multipliers. Colloq. Math. 127, 17-37, 2012.

[BCR] C. Berg, J. Christensen and P. Ressel. Harmonic analysis on semigroups. Theory of positive definite and related functions. Springer-Verlag, New York, 1984.

[BDJ] N. Brown, K. Dykema and K. Jung. Free entropy dimension in amalgamated free products. With an appendix by Wolfgang Lück. Proc. Lond. Math. Soc. 97, no. 2, 339-367, 2008.

[BeL] J. Bergh and J. Löfström. Interpolation spaces. Springer-Verlag, Berlin, 1976.

[BGM] E. Berkson, T. Gillespie and P. Muhly. Generalized analyticity in UMD spaces. Ark. Mat. 27, no. 1: 1-14, 1989.

[BiS] M. Birman and M. Solomyak. Double operator integrals in a Hilbert space. Integral Equations Operator Theory 47, no. 2: 131-168, 2003.

[BKS] M. Bożejko, B. Kümmerer and R. Speicher. q-Gaussian processes: non-commutative and classical aspects. Comm. Math. Phys. 185, no. 1, 129-154, 1997.

[Bla] B. Blackadar. Operator algebras. Theory of $C^{*}$-algebras and von Neumann algebras. Springer-Verlag, Berlin, 2006.

[BID] E. Blanchard and K. Dykema. Embeddings of reduced free products of operator algebras.. Pacific J. Math. 199, no. 1: 1-19, 2001.

[BoS] M. Bożejko and R. Speicher. Completely positive maps on Coxeter groups, deformed commutation relations, and operator spaces. Math. Ann. 300, no. 1: 97-120, 1994.

[Boz] M. Bożejko. Bessis-Moussa-Villani conjecture and generalized Gaussian random variables. Infin. Dimens. Anal. Quantum Probab. Relat. Top. 11, no. 3: 313-321, 2008.

[Cha] Chatterji, S. D. Martingale convergence and the Radon-Nikodym theorem in Banach spaces. Math. Scand. 22, 21-41, 1968.

[Con] A. Connes. Classification of injective factors. Ann. of Math. 104, no. 1: 73-115, 1976.

[CoW] R. Coifman and G. Weiss. Transference methods in analysis. Conference Board of the Mathematical Sciences Regional Conference Series in Mathematics, No. 31. American Mathematical Society, Providence, R.I., 1976.

[DCH] J. De Cannière and U. Haagerup. Multipliers of the Fourier algebras of some simple Lie groups and their discrete subgroups. Amer. J. Math. 107, no. 2: 455-500, 1985. 
[DeL] K. De Leeuw. On $L_{p}$ multipliers. Ann. of Math. 81: 364-379, 1965.

[DiU] J. Diestel and J. Uhl. Vector measures. American Mathematical Society, Providence, 1977.

[Dr] S. W. Drury. A counterexample to a conjecture of Matsaev. Lin. Alg. and its Appl. 435, 323-329 (2011).

[EfR] E. Effros and Z-J. Ruan. Operator spaces. Oxford University Press, 2000.

[EfP] E. Effros and M. Popa. Feynman diagrams and Wick products associated with q-Fock space. Proc. Natl. Acad. Sci. USA 100, no. 15: 8629-8633, 2003.

[Fen] G. Fendler. Dilations of one parameter semigroups of positive contractions on $L^{p}$ spaces. Canad. J. Math. 49, no. 4: 736-748, 1997.

[Jod] M. Jodeit. Restrictions and extensions of Fourier multipliers. Studia Math. 34: 215-226, 1970.

[JMX] M. Junge, C. Le Merdy and Q. Xu. $H^{\infty}$ functional calculus and square functions on noncommutative $L^{p}$-spaces. Astérisque, no. 305, 2006.

[JLM] M. Junge and C. Le Merdy. Dilations and rigid factorisations on noncommutative $L_{p}$-spaces. J. Funct. Anal. 249: 220-252, 2007.

[JuX] M. Junge and Q. Xu. Noncommutative maximal ergodic theorems. J. Amer. Math. Soc. 20, no. 2: 385-439, 2007.

[Jun] M. Junge. Fubini's theorem for ultraproducts of noncommmutative $L_{p}$-spaces II. Preprint.

[Kir] E. Kirchberg. On nonsemisplit extensions, tensor products and exactness of group $\mathrm{C}^{*}$-algebras. Invent. Math. 112, no. 3: 449-489, 1993.

[Kit] A. Kitover. A question in connection with Matsaev's conjecture. Linear and Complex Analysis Problem Book 3, Part I, Lecture Notes in Math. 1573: 247, Springer, Berlin, 1985.

[Lar] R. Larsen. An introduction to the theory of multipliers. Springer-Verlag, 1971.

[LaS] V. Lafforgue and M. de la Salle. Noncommutative $L^{p}$-spaces without the completely bounded approximation property. Duke Math. J. 160 , no. 1, 71-116, 2011.

[MeN] P. Meyer-Nieberg. Banach lattices. Springer-Verlag, Berlin, 1991.

[NeR] S. Neuwirth and E. Ricard. Transfer of Fourier multipliers into Schur multipliers and sumsets in a discrete group. Canad. J. Math. 63, no. 5, 1161-1187, 2011.

[Nik1] N. K. Nikolski. Five problems on invariant subspaces. J. Sov. Math., 2: 441-450, 1974.

[Nik2] N. Nikolski. Operators, functions, and systems: an easy reading, vol 2. American Mathematical Society, 2002.

[Nou] A. Nou. Asymptotic matricial models and QWEP property for $q$-Araki-Woods algebras. J. Funct. Anal. 232, no. 2: 295-327, 2006.

[Oza] N. Ozawa. About the QWEP conjecture. Internat. J. Math. 15, no. 5: 501-530, 2004.

[Pau] V. Paulsen. Completely bounded maps and operator algebras. Cambridge Univ. Press, 2002.

[Pel1] V.V. Peller. An Analogue of an inequality of J. von Neumann, isometric dilation of contractions, and approximation by isometries in spaces of measurable functions. Proc. Steklov Inst. Math. 1 : 101-145, 1983.

[Pel2] V.V. Peller. Estimates of operator polynomials on the Schaten - von Neumann classes. Linear and Complex Analysis Problem Book 3, Part I, Lecture Notes in Math. 1573: 244-246, Springer, Berlin, 1985.

[Pis1] G. Pisier. Regular operators between non-commutative $L_{p}$-spaces. Bull. Sci. Math. 119 : 95-118, 1995.

[Pis2] G. Pisier. Non-commutative vector valued $L_{p}$-spaces and completely p-summing maps. Astérisque, 247, 1998.

[Pis3] G. Pisier. Similarity problems and completely bounded maps, volume 1618 of Lecture Notes in Mathematics. Springer-Verlag, expanded edition, 2001.

[Pis4] G. Pisier. Introduction to operator space theory. Cambridge University Press, Cambridge, 2003. 
[PiX] G. Pisier and Q. Xu. Non-commutative $L_{p}$-spaces. Handbook of the Geometry of Banach Spaces volume II: 1459-1517, 2003.

[Ric] E. Ricard. A Markov dilation for self-adjoint Schur multipliers. Proc. Amer. Math. Soc. 136: 4365-4372, 2008.

[Sak] S. Sakai. $C^{*}$-algebras and $W^{*}$-algebras. Springer-Verlag, New York, 1971.

[Sun] V. Sunder. An invitation to von Neumann algebras. Springer-Verlag, New York, 1987.

[SNF] B. Sz.-Nagy and C. Foias. Harmonic analysis of operators on Hilbert space. North-Holland, AmsterdamLondon, 1970.

[Str] S. Stratila. Modular theory in operator algebras. Taylor and Francis, 1981.

[Tak3] M. Takesaki. Theory of operator algebras. III. Springer-Verlag, Berlin, 2003.

[Ued] Y. Ueda. Amalgamated free product over Cartan subalgebra. Pacific J. Math. 191, no. 2: 359-392, 1999.

Laboratoire de Mathématiques, Université de Franche-Comté, 25030 Besançon Cedex, France cedric.arhancet@univ-fcomte.fr 